Internet Engineering Task Force (IETF)

E. Beili

Request for Comments: 6765

Category: Standards Track

ISSN : $2070-1721$

Actelis Networks

M. Morgenstern

ECI Telecom

February 2013

xDSL Multi-Pair Bonding (G.Bond) MIB

Abstract

This document defines a Management Information Base (MIB) module for use with network management protocols in TCP/IP-based internets.

This document defines an extension to the Interfaces Group MIB with a set of common objects for managing multi-pair bonded Digital

Subscriber Line (xDSL) interfaces, as defined in ITU-T

Recommendations G.998.1, G.998.2, and G.998.3. The textual

conventions defining the bonding schemes are contained in a separate MIB module maintained by Internet Assigned Numbers Authority (IANA) . The MIB modules specific to each bonding technology are defined in G9981-MIB, G9982-MIB, and G9983-MIB, respectively.

Status of This Memo

This is an Internet Standards Track document.

This document is a product of the Internet Engineering Task Force (IETF). It represents the consensus of the IETF community. It has received public review and has been approved for publication by the Internet Engineering Steering Group (IESG). Further information on Internet Standards is available in Section 2 of RFC 5741.

Information about the current status of this document, any errata, and how to provide feedback on it may be obtained at http://www.rfc-editor.org/info/rfc6765. 
Copyright Notice

Copyright (c) 2013 IETF Trust and the persons identified as the document authors. All rights reserved.

This document is subject to BCP 78 and the IETF Trust's Legal Provisions Relating to IETF Documents

(http://trustee.ietf.org/license-info) in effect on the date of publication of this document. Please review these documents carefully, as they describe your rights and restrictions with respect to this document. Code Components extracted from this document must include Simplified BSD License text as described in Section 4.e of the Trust Legal Provisions and are provided without warranty as described in the Simplified BSD License.

Table of Contents

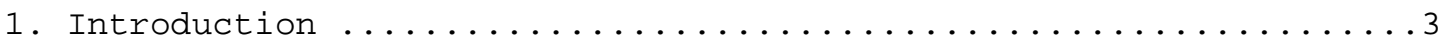

2. The Internet-Standard Management Framework .................

3. The Broadband Forum Management Framework for xDSL Bonding ......4

4. Relationship to Other MIB Modules .....................

4.1. Relationship to Interfaces Group MIB Module ..............

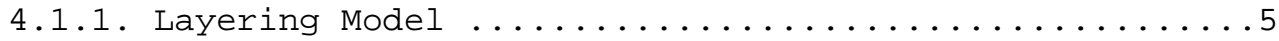

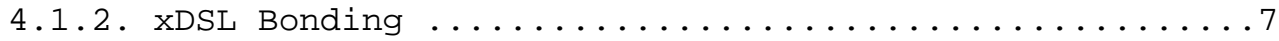

4.1.3. Discovery Operation .........................

4.1.4. Initialization of G.Bond Ports ..............

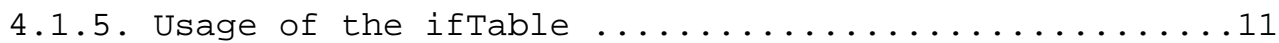

4.2. Relationship to G.Bond ATM, ETH, and TDIM MIB Modules ....13

4.3. Relationship to xDSL MIB Modules ............................

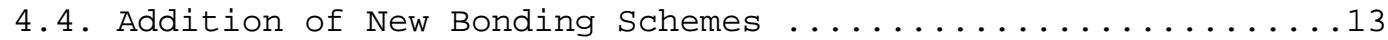

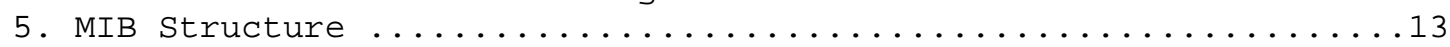

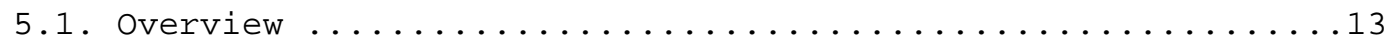

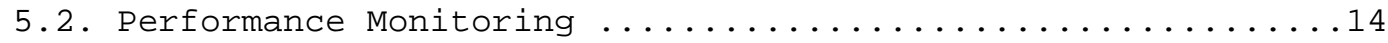

5.3. Mapping of Broadband Forum TR-159 Managed Objects ........14

6. xDSL Multi-Pair Bonding MIB Definitions ...................

7. IANA-Maintained G.Bond TC Definitions ..................65

8. Security Considerations ........................67

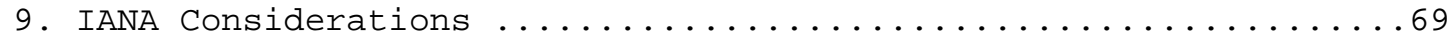

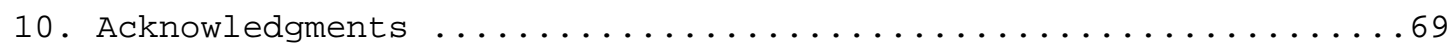

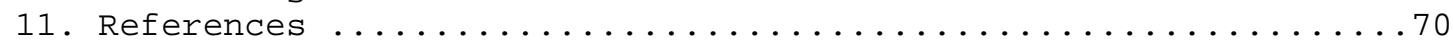

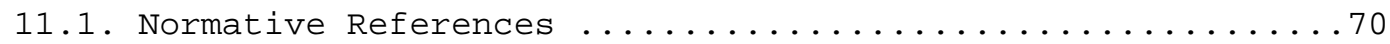

11.2. Informative References ........................ 


\section{Introduction}

xDSL Multi-pair bonding allows a service provider to provide highbandwidth services to business and residential customers over multiple xDSL lines, with greater speed and resiliency than service over a single xDSL line, bridging the gap between xDSL and fiberbased transport.

Currently, there are three xDSL Multi-pair bonding schemes, also known under the collective name "G.Bond":

- ATM-Based Multi-pair bonding, as specified in ITU-T Recommendation G.998.1 [G.998.1], which defines a method for the bonding (or aggregating) of multiple xDSL lines (or individual bearer channels in multiple xDSL lines) into a single bidirectional logical link carrying an ATM stream. This specification can be viewed as an evolution of the legacy Inverse Multiplexing for ATM (IMA) technology [AF-PHY-0086], applied to xDSL with variable rates on each line/bearer channel.

- Ethernet-Based Multi-pair bonding, as specified in ITU-T Recommendation G.998.2 [G.998.2], which defines a method for the bonding (or aggregating) of multiple xDSL lines (or individual bearer channels in multiple xDSL lines) into a single bidirectional logical link carrying an Ethernet stream. This specification can be viewed as IEEE 802.3-2005 [802.3] Clause 61, Physical Medium Entity (PME) Aggregation, generalized to work over any xDSL technology (2Base-TL and 10Pass-TS interfaces defined by IEEE use G.SHDSL (Single-pair High-speed DSL) and VDSL (Very high speed DSL) technology, respectively).

- Multi-pair bonding using Time-Division Inverse Multiplexing (TDIM), specified in ITU-T Recommendation G.998.3 [G.998.3], which defines a method for the bonding (or aggregating) of multiple xDSL lines into a single bidirectional logical link carrying a mix of various traffic streams (e.g., Ethernet, ATM, TDM).

Architecturally, all three bonding schemes define a new "bonded" Transport Protocol Specific - Transmission Convergence (TPS-TC) sub-layer, stacked above multiple ATM-TC, Ethernet/Packet Transfer Mode-TC (PTM-TC), or Synchronous Transfer Mode-TC (STM-TC) (clear channel) sub-layers for the ATM, Ethernet, or TDIM bonding, respectively. Each underlying TPS-TC sub-layer represents a protocol-specific interface to an xDSL line or an individual bearer channel of an xDSL line. Bonding of multiple bearer channels in the same xDSL line is not allowed. 
All schemes allow bonding of up to 32 individual line/channel sub-layers with variable rates, providing common functionality for the configuration, initialization, operation, and monitoring of the bonded link.

This document defines a MIB module common to all 3 schemes. Additional managed objects specific to each bonding technology are defined in the G9981-MIB [RFC6768], G9982-MIB [RFC6767], and G9983-MIB [RFC6766] modules.

The textual conventions listing the bonding schemes are defined in a separate, IANA-maintained MIB module, the first version of which is provided in this document. This arrangement would allow for future bonding schemes to be easily supported, without the need to update the common GBOND-MIB module.

2. The Internet-Standard Management Framework

For a detailed overview of the documents that describe the current Internet-Standard Management Framework, please refer to section 7 of RFC 3410 [RFC3410].

Managed objects are accessed via a virtual information store, termed the Management Information Base or MIB. MIB objects are generally accessed through the Simple Network Management Protocol (SNMP). Objects in the MIB are defined using the mechanisms defined in the Structure of Management Information (SMI). This document specifies a MIB module that is compliant to the SMIV2, which is described in STD 58, RFC 2578 [RFC2578], STD 58, RFC 2579 [RFC2579] and STD 58, RFC 2580 [RFC2580].

The key words "MUST", "MUST NOT", "REQUIRED", "SHALL", "SHALL NOT", "SHOULD", "SHOULD NOT", "RECOMMENDED", "NOT RECOMMENDED", "MAY", and "OPTIONAL" in this document are to be interpreted as described in BCP 14, RFC 2119 [RFC2119].

3. The Broadband Forum Management Framework for xDSL Bonding

This document makes use of the Broadband Forum technical report "Management Framework for xDSL Bonding" [TR-159], defining a management model and a hierarchy of management objects for the bonded xDSL interfaces. 


\section{Relationship to Other MIB Modules}

This section outlines the relationship of the MIB modules defined in this document with other MIB modules described in the relevant RFCs. Specifically, the following MIB modules are discussed: the Interfaces Group MIB (IF-MIB), Inverse Stack Table MIB (IF-INVERTED-STACK-MIB), and Interface Stack Capability MIB (IF-CAP-STACK-MIB); G.Bond schemespecific modules G.Bond/ATM (G9981-MIB), G.Bond/Ethernet (G9982-MIB), and G.Bond/TDIM (G9983-MIB); and DSL-specific MIB modules ADSL (ADSL-LINE-EXT-MIB), ADSL2 (ADSL2-LINE-MIB), SHDSL (HDSL2-SHDSL-LINE-MIB), VDSL (VDSL-LINE-MIB), and VDSL2 (VDSL2-LINE-MIB) .

\subsection{Relationship to Interfaces Group MIB Module}

A bonded xDSL port is a stacked (a.k.a. aggregated or bonded) interface and as such is managed using generic interface management objects defined in the IF-MIB [RFC2863].

The stack management, i.e., actual connection of the sub-layers to the top layer interface, is done via the ifstackTable, as defined in the IF-MIB [RFC2863] and its inverse -- the ifInvStackTable, as defined in the IF-INVERTED-STACK-MIB [RFC2864].

The ifCapstackTable and its inverse -- the ifInvCapstackTable, as defined in the IF-CAP-STACK-MIB [RFC5066] -- extend the stack management with an ability to describe possible connections or crossconnect capability, when a flexible cross-connect matrix is present between the interface layers.

\subsubsection{Layering Model}

A G.Bond interface can aggregate up to 32 channel sub-layers, with each channel representing an xDSL line or an xDSL bearer channel. For the purpose of brevity we will refer to the bonded interface as the Generic Bonding Sub-layer (GBS) and to the channel sub-layer as the Bonding Channel Entity (BCE) .

A generic G.Bond device can have a number of GBS ports, each connected to a particular upper layer (e.g., a Media Access Control (MAC) interface for the G.998.2 scheme), while simultaneously crossconnected to a number of underlying BCEs, with a single-GBS-per-BCE relationship.

A GBS port is represented in the Interfaces table (ifTable) as a separate interface with an iftype reflecting a particular bonding scheme, e.g., g9981(263), g9982(264), or g9983(265). 
Each BCE in the aggregated GBS port is represented in the ifTable as a separate interface with an iftype relevant to a particular xDSL technology, e.g., shdsl(169) or vdsl(97). The iftype values are defined in [IANAifType-MIB].

The following figure shows the layering diagram and corresponding use of the ifTable for the bonded xDSL interfaces:
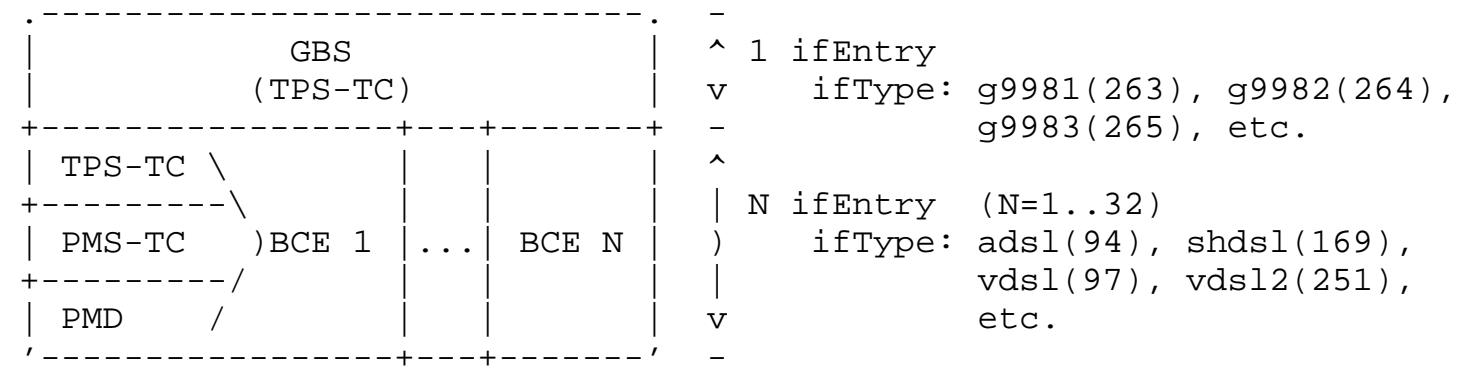

The ifstackTable is indexed by the ifIndex values of the aggregated G.Bond port (GBS) and the BCEs connected to it. The ifstackTable allows a network management application to determine which BCEs are connected to a particular GBS and change connections (if supported by the application). The ifInvstackTable, being an inverted version of the ifstackTable, provides an efficient means for a network management application to read a subset of the ifStackTable and thereby determine which GBS runs on top of a particular BCE.

The ifCapstackTable, defined in the IF-CAP-STACK-MIB module, specifies for each higher-layer interface (e.g., GBS port) a list of lower-layer interfaces (e.g., BCEs), which can possibly be crossconnected to that higher-layer interface, determined by the crossconnect capability of the device. This table, modeled after the ifstackTable, is read only, reflecting current cross-connect capability of a stacked interface, which can be dynamic in some implementations (e.g., if xDSL lines are located on a pluggable module and the module is pulled out). Note that BCE availability per GBS, described by the ifcapstackTable, can be constrained by other parameters -- for example, by the aggregation capacity of a GBS or by the $\mathrm{BCE}$ in question being already connected to another GBS. So, in 
order to ensure that a particular BCE can be connected to the GBS, all respective parameters (e.g., ifCapstackTable, ifstackTable, and gBondPortCapCapacity) SHALL be inspected.

The ifInvCapStackTable, also defined in the IF-CAP-STACK-MIB module, describes which higher-layer interfaces (e.g., GBS ports) can possibly be connected to a particular lower-layer interface (e.g., BCE), providing inverted mapping of the ifCapstackTable. While it contains no additional information beyond that already contained in the ifCapstackTable, the ifInvCapstackTable has the ifIndex values in its INDEX clause in the reverse order, i.e., the lower-layer interface first, and the higher-layer interface second, providing efficient means for a network management application to read a subset of the ifCapstackTable and thereby determine which interfaces can be connected to run on top of a particular interface.

\subsection{2. xDSL Bonding}

The G.998.x Bonding allows a number of BCEs to be aggregated onto a single logical GBS port by splitting the incoming traffic into multiple streams, transmitting each stream over a specific BCE, and combining the streams at the remote GBS port, preserving the original traffic order.

The Ethernet frames MAY be fragmented before the transmission and reassembled at the remote end to minimize transportation delay. The G.998.2 (G.Bond/Ethernet) ports with multiple BCEs MUST perform the fragmentation and reassembly of the Ethernet frames. However, for single-BCE G.998.2 ports this function MAY be omitted (a.k.a. bonding bypass), to minimize fragmentation overhead and additional processing delay as well as to be able to interoperate with non-G.998 DSL equipment.

The agent is REQUIRED to indicate all supported bonding schemes (for example, ATM, Ethernet, and TDIM), including OPTIONAL support for the bonding bypass in G.998.2 single-BCE ports.

The GBOND-MIB module allows a network management application to query Bonding capability and enable/disable it if supported. Note that enabling Bonding (by setting the value of the gBondPortConfAdminScheme and gBondPortConfPeerAdminScheme objects to any supported bonding scheme other than 'none') effectively turns on the fragmentation and reassembly function, even on a single-BCE port. 


\subsubsection{Discovery Operation}

The G.Bond ports may optionally support a discovery operation whereby BCEs, during initialization, exchange information about their respective aggregation groups (GBS), via the [G.994.1] handshake (G.hs) protocol. This information can then be used to detect copper misconnections or for an automatic assignment of the local BCEs into aggregation groups instead of a fixed preconfiguration.

The MIB module defined in this document allows a network management application to control the G.Bond discovery mechanism and query its results.

Two tables are used by the G.Bond discovery mechanism: the ifstackTable and the ifcapstackTable. The following pseudocode gives an example of the discovery and automatic BCE assignment for a generic multi-GBS G.Bond device, located at the Central Office (CO), using objects defined in this MIB module as well as the IF-CAP-STACK-MIB and IF-MIB modules [Note that automatiC BCE assignment is only shown here for the purposes of the example. Fixed BCE pre-assignment, manual assignment, or auto-assignment using an alternative internal algorithm may be chosen by a particular implementation ] :

// Go over all GBS ports in the CO device FOREACH gbs[i] IN CO_device

\{ // Perform discovery and auto-assignment on GBS ports

// with room for more channels.

IF ( gbs [i]. NumBCEs < gbs [i]. BondCapacity)

\{ // Assign a unique 6-octet local discovery code to the GBS,

// e.g., MAC address of the associated port or some other

// unique number specifically allocated for this purpose.

$\mathrm{dc}=$ gbs [i]. DiscoveryCode = MAC[i];

// Go over all disconnected channels, which can

// potentially be connected to the GBS.

FOREACH bce[j] IN ifCapstackTable[gbs[i]] AND

NOT IN ifstackTable[gbs[i]] // not connected

\{ // Try to grab the Remote Terminal device (RT) by writing the

// value of the local 6-byte discovery code to the remote

// discovery code register (via a handshake mechanism).

// This operation is an atomic Set-if-Clear action; i.e., it

// would succeed only if the remote discovery register was

// zero. Read the remote discovery code register via a Get

// operation to see if the RT, attached via the $\mathrm{BCE}$,

// is indeed marked as being the CO_device peer.

bce [j].RemoteDiscoverycode = dc; // Set-if-clear

$r=$ bce $[j]$. RemoteDiscoveryCode;

// Get 


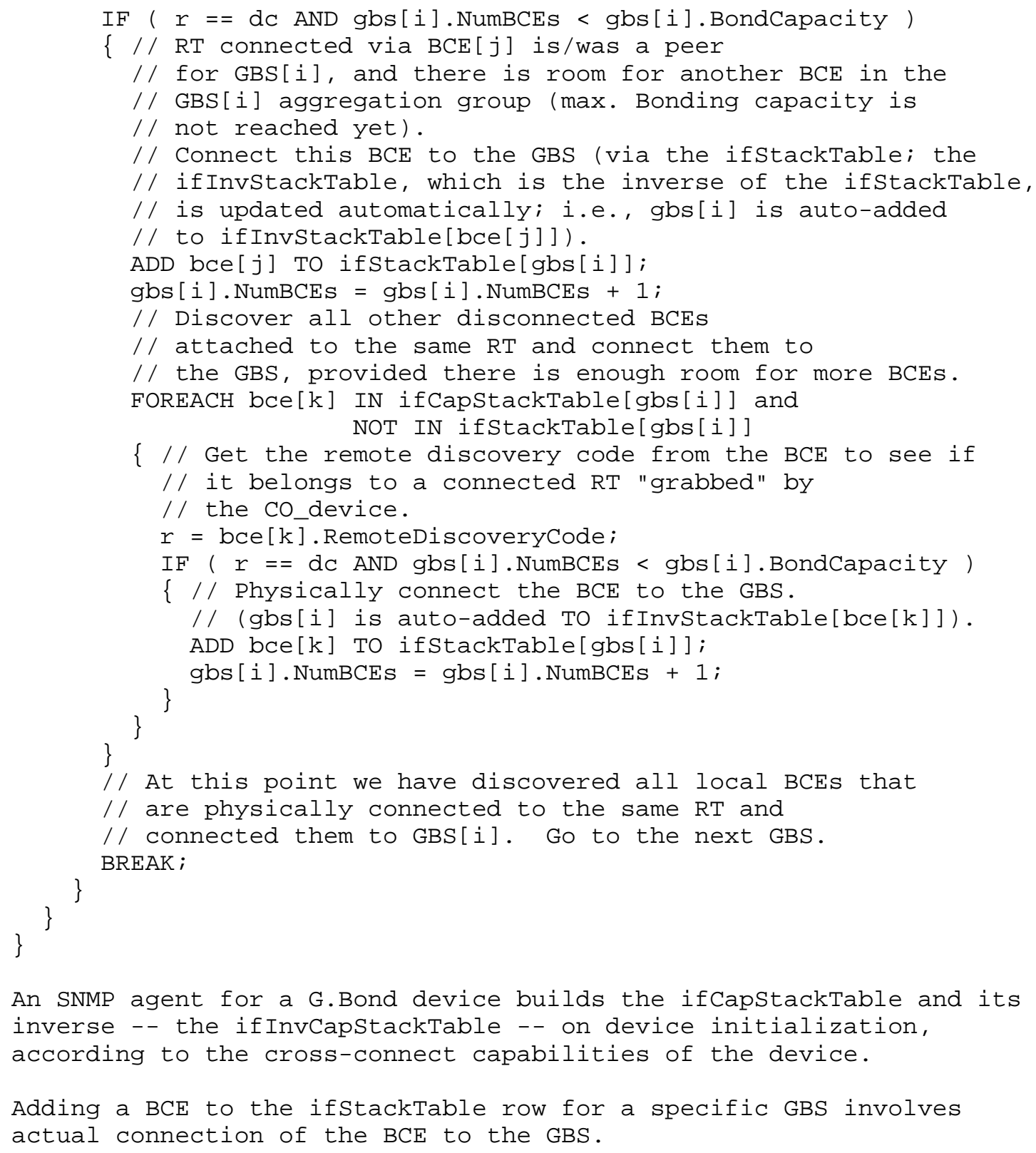

An SNMP agent for a G.Bond device builds the ifCapstackTable and its inverse -- the ifInvCapStackTable -- on device initialization, according to the cross-connect capabilities of the device.

Adding a BCE to the ifstackTable row for a specific GBS involves actual connection of the BCE to the GBS. 
Note that a GBS port does not have to be operationally 'down' for the connection to succeed. In fact, a dynamic BCE addition (and removal) MAY be implemented with an available BCE being initialized first (by setting its ifAdminstatus to 'up') and then added to an operationally 'up' GBS port, by modifying a respective ifstackTable (and respective ifInvStackTable) entry.

It is RECOMMENDED that removal of the last operationally 'up' BCE from an operationally 'up' GBS, i.e., modification of a respective entry in the ifstackTable, and a corresponding entry in the ifInvstackTable, would be rejected by the implementation (in the case of SNMP, with the error inconsistentValue), as this action would completely drop the link.

In addition to the standard handshake-based discovery described above, [G.998.2] defines an optional frame-based discovery and pair management. These frame-based methods are discussed in [RFC6767].

\subsubsection{Initialization of G.Bond Ports}

G.Bond ports built on top of xDSL technology require a lengthy initialization or 'training' process before any data can pass. During this initialization, both ends of a link (peers) work cooperatively to achieve a required data rate on a particular copper pair. Sometimes, when the copper line is too long or the noise on the line is too high, that 'training' process may fail to achieve a specific target rate with required characteristics.

The ifAdminstatus object from the IF-MIB controls the desired state of a GBS with all the BCEs connected to it or of an individual BCE port. Setting this object to 'up' instructs a particular GBS or a $\mathrm{BCE}$ to start the initialization process, which may take tens of seconds for G.Bond ports. The ifoperstatus object from the IF-MIB shows the operational state of an interface for the GBS, extended by the gBondPortstatfltstatus object defined in this document, and a corresponding * Status object from a relevant xDSL line MIB for BCE interfaces.

A disconnected BCE may be initialized by changing the ifAdminstatus from 'down' to ' up'. Changing the ifAdminstatus to 'up' on the GBS initializes all BCEs connected to that particular GBS. Note that in the case of bonding, some interfaces may fail to initialize while others succeed. The GBS is considered operationally 'up' if at least one bonded BCE is operationally 'up'. When all BCEs connected to the GBS are 'down', the GBS SHALL be considered operationally

'lowerLayerDown'. The GBS SHALL be considered operationally 
'notPresent' if it is not connected to any BCE. The GBS/BCE

interface SHALL remain operationally 'down' during initialization, indicated by the 'init' value of the gBondPortstatfltstatus object.

4.1.5. Usage of the iftable

Both BCE and GBS interfaces are managed using interface-specific management objects defined in the GBOND-MIB module and generic interface objects from the ifTable of the IF-MIB, with all management table entries referenced by the interface index ifIndex. 
The following table summarizes G.Bond-specific interpretations for some of the iftable objects specified by the mandatory ifGeneralinformationGroup:

\begin{tabular}{|c|c|}
\hline IF-MIB Object & G.Bond Interpretation \\
\hline ifIndex & $\begin{array}{l}\text { Interface index. Note that each BCE and each GBS } \\
\text { in the G.Bond PHY MUST have a unique index, as } \\
\text { there are some GBS- and BCE-specific attributes } \\
\text { accessible only on the GBS or BCE level. }\end{array}$ \\
\hline iftype & $\begin{array}{l}\text { g9981 (263), g9982(264), or } 99983(265) \text { for the } \\
\text { ATM, Ethernet, or TDIM GBS, respectively; } \\
\text { shdsl(169) for the G.SHDSL BCE, vdsl(97) for the } \\
\text { VDSL BCE, etc. }\end{array}$ \\
\hline ifspeed & $\begin{array}{l}\text { Operating data rate for the BCE. For the GBS, it } \\
\text { is the sum of the current operating data rates of } \\
\text { all BCEs in the aggregation group, without the } \\
\text { encapsulation overhead and G.Bond overhead, but } \\
\text { accounting for Inter-Frame Gaps (IFG). When a } \\
\text { GBS or a BCE is operating in an asymmetrical } \\
\text { fashion (the upstream data rate differs from the } \\
\text { downstream one), the lowest of the values is } \\
\text { shown. }\end{array}$ \\
\hline ifAdminstatus & $\begin{array}{l}\text { Setting this object to 'up' instructs a } \\
\text { particular GBS (with all BCEs connected to it) or } \\
\text { a BCE to start the initialization process. }\end{array}$ \\
\hline ifoperstatus & $\begin{array}{l}\text { A relevant *Status object from a particular line } \\
\text { MIB supplements the value of ifoperStatus for } \\
\text { BCEs. gBondPortstatFltStatus supplements the } \\
\text { value of ifoperStatus for a GBS. Note that both } \\
\text { relevant objects shall be inspected to determine } \\
\text { the real operational status of a BCE/GBS port, } \\
\text { e.g., a GBS port may be operationally ' up' with } \\
\text { gBondPortStatFltstatus indicating lowRate(4) } \\
\text { fault condition, or 'down' with no gBond faults. }\end{array}$ \\
\hline
\end{tabular}

Table 1: G.Bond Interpretation of IF-MIB Objects 
4.2. Relationship to G.Bond ATM, ETH, and TDIM MIB Modules

The MIB module defined in this document is common to all G.998 bonding schemes. It MUST be used in conjunction with a bonding scheme-specific MIB module:

o G9981-MIB [RFC6768] for a G.998.1 bonded interface.

- G9982-MIB [RFC6767] for a G.998.2 bonded interface.

o G9983-MIB [RFC6766] for a G.998.3 bonded interface.

4.3. Relationship to xDSL MIB Modules

Each xDSL technology is described in a relevant xDSL line MIB module: e.g., the HDSL2-SHDSL-LINE-MIB [RFC4319] for G.SHDSL, ADSL-LINE-EXT-MIB [RFC3440] for ADSL, ADSL2-LINE-MIB [RFC4706] for ADSL2, VDSL-LINE-MIB [RFC3728] for VDSL, or VDSL2-LINE-MIB [RFC5650] for VDSL2.

These MIB modules are used to manage individual xDSL lines/channels (BCES) .

4.4. Addition of New Bonding Schemes

In case a new bonding scheme is introduced in a revision of G.998, IANA can update the IANA-maintained MIB module, adding the corresponding new value to the IANAgBondScheme and IANAgBondSchemelist textual conventions, as well as listing the new scheme-specific MIB module's name (e.g., G998x-MIB).

Any scheme-specific aspect of an existing GBOND-MIB object SHALL be described in the corresponding G998x-MIB module, to prevent an unnecessary reissue of the GBOND-MIB module. For example, an exact definition of an Errored Second (ES) or a Severely Errored Second (SES) can be bonding-scheme specific; see the definitions for the gBondPortPmCurES and gBondPortPmCurSES objects.

5. MIB structure

\subsection{Overview}

The main management objects defined in the GBOND-MIB module are split into 2 groups, structured as recommended by RFC 4181 [RFC4181]:

- gBondPort - containing objects for configuration, capabilities, status, historical Performance Monitoring, and notifications, common to all G.Bond ports (GBS). 
- gBondBce - containing a single common object for configuration of the remote discovery code per BCE. Note that the rest of the objects for BCE configuration, capabilities, status, and notifications are located in relevant XDSL line MIB modules as well as in the bonding scheme-specific MIB modules.

\subsection{Performance Monitoring}

The OPTIONAL Performance Monitoring counters, thresholds, and history buckets (interval-counters) defined in [TR-159] are implemented using the textual conventions defined in the HC-PerfHist-TC-MIB [RFC3705]. The HC-PerfHist-TC-MIB defines 64-bit versions of the textual conventions found in the PerfHist-TC-MIB [RFC3593].

The agent SHOULD align the beginning of each interval to a fifteenminute boundary of a wall clock. Likewise, the beginning of each one-day interval SHOULD be aligned with the start of a day.

The rationale behind this is to simplify collection and analysis of Performance Monitoring (PM) from multiple agents by a network management system (NMS) -- each PM interval can be "time-stamped" using the gBond*Intervalindex object, from the fact that the 1-day interval starts at 00:00 and the 15-minute intervals are aligned with each 1/4 hour and the network-wide "wall clock", typically distributed via NTP or the Simple Network Time Protocol (SNTP) [RFC5905]. If the agent does not have access to the wall clock, a local clock can be used. In this case, as well as when coping with multiple time zones, the NMS would have to correlate the difference between the agent's local clock (available, for example, via the hrSystemDate object from the HOST-RESOURCES-MIB [RFC2790]) and the wall clock.

Counters are not reset when a GBS is re-initialized, but rather only when the agent is reset or re-initialized.

Note that the accumulation of certain performance events for a monitored entity is inhibited (counting stops) during periods of service unavailability on that monitored entity. The DESCRIPTION clause of Performance Monitoring counters in this MIB module specifies which of the counters are inhibited during periods of service unavailability.

\subsection{Mapping of Broadband Forum TR-159 Managed Objects}

This section contains the mapping between relevant managed objects (attributes) defined in [TR-159] and managed objects defined in this document and in associated MIB modules, i.e., the IF-MIB [RFC2863]. 


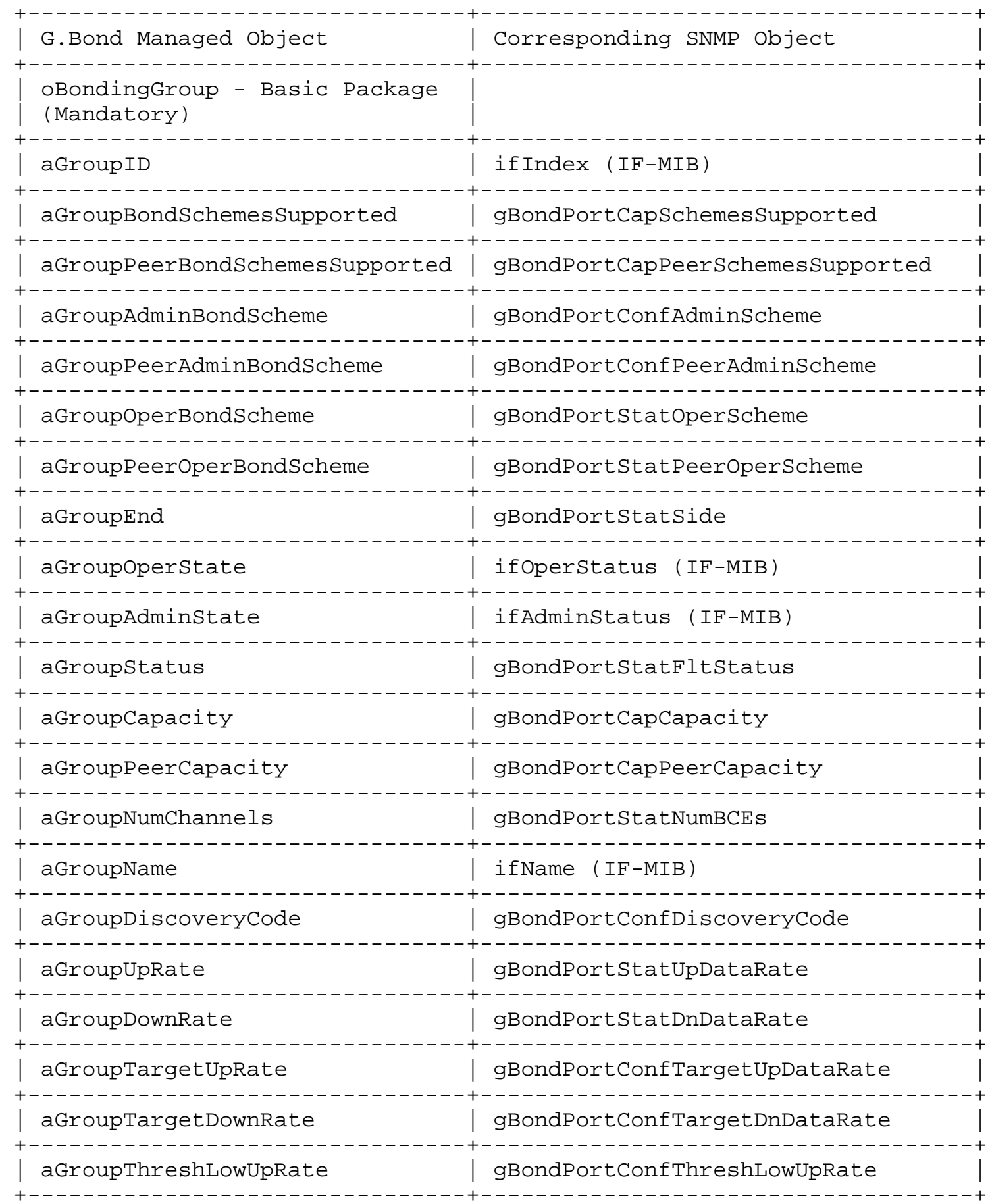




\begin{tabular}{|c|c|}
\hline aGroupThreshLowDownRate & gBondPortConfThreshLowDnRate \\
\hline aGroupLowRateCrossingEnable & gBondPortConfLowRateCrossingEnable \\
\hline------------------------- & ------------------------ \\
\hline nGroupLowUpRateCrossing & gBondLowUpRateCrossing \\
\hline nGroupLowDownRateCrossing & gBondLowDnRateCrossing \\
\hline aGroupLinkUpDownEnable & ifLinkUpDownTrapEnable (IF-MIB) \\
\hline nGroupLinkUp & linkUp (IF-MIB) \\
\hline nGroupLinkDown & linkDown (IF-MIB) \\
\hline $\begin{array}{l}\text { oBondingGroup - PM Package } \\
\text { (Optional) }\end{array}$ & \\
\hline aGroupPerfes & gBondPortPmCurES \\
\hline aGroupPerfSES & gBondPortPmCurSES \\
\hline aGroupPerfUAS & gBondPortPmCurUAS \\
\hline aGroupPerf15MinValidIntervals & gBondPortPmCur15MinValidIntervals \\
\hline aGroupPerf15MinInvalidIntervals & gBondPortPmCur15MinInvalidIntervals \\
\hline aGroupPerfCurr15MinTimeElapsed & gBondPortPmCur15MinTimeElapsed \\
\hline aGroupPerfCurr15MinES & $\begin{array}{l}\text { gBondPortPmCur15MinES } \\
\text { M PM }\end{array}$ \\
\hline--------------------------1 & --------------------------- \\
\hline aGroupPerfCurr15MinSES & gBondPortPmCur15MinSES \\
\hline aGroupPerfCurr15MinUAS & gBondPortPmCur15MinUAS \\
\hline aGroupPerfTcaEnable & gBondPortConfPmTcaEnable \\
\hline aGroupPerfThreshold15MinES & gBondPortPmTcaProfileThresh15MinES \\
\hline aGroupPerfThreshold15MinSES & gBondPortPmTcaProfileThresh15MinSES| \\
\hline------------------------------- & ------------------------------------+ \\
\hline aGroupPerfThreshold15MinUAS & gBondPortPmTcaProfileThresh15MinUAS \\
\hline nGroupPerfTca15MinES & gBondPmTca15MinESCrossing \\
\hline SES & sing \\
\hline
\end{tabular}




\begin{tabular}{|c|c|}
\hline nGroupPerfTca15MinUAS & gBondPmTca15MinUASCrossing \\
\hline aGroupPerf1DayValidIntervals & gBondPortPmCur1DayValidIntervals \\
\hline- & -- \\
\hline aGroupPerf1DayInvalidIntervals & gBondPortPmCur1DayInvalidIntervals \\
\hline aGroupPerfCurr1DayTimeElapsed & gBondPortPmCur1DayTimeElapsed \\
\hline aGroupPerfCurr1DayES & gBondPortPmCur1DayIntervalES \\
\hline aGroupPerfCurr1DaysES & gBondPortPmCur1DayIntervalSES \\
\hline--------------------------- & ------------ \\
\hline aGroupPerfCurr1DayUAS & gBondPortPmCur1DayIntervalUAS \\
\hline aGroupPerfThreshold1DayES & gBondPortPmTcaProfileThresh1DayES \\
\hline aGroupPerfThreshold1DaySES & gBondPortPmTcaProfileThresh1DaySES \\
\hline aGroupPerfThreshold1DayUAS & gBondPortPmTcaProfileThresh1DayUAS \\
\hline nGroupPerfTca1DayES & gBondPmTca1DayESCrossing \\
\hline nGroupPerfTcalDaySES & gBondPmTcalDaySESCrossing \\
\hline nGroupPerfTca1DayUAS & gBondPmTca1DayUASCrossing \\
\hline aGroupPerf15MinIntervalNumber & gBondPortPm15MinIntervalIndex \\
\hline aGroupPerf15MinIntervalValid & gBondPortPm15MinIntervalValid \\
\hline aGroupPerf15MinIntervalES & gBondPortPm15MinIntervalES \\
\hline aGroupPerf15MinIntervalSES & gBondPortPm15MinIntervalSES \\
\hline aGroupPerf15MinIntervalUAS & gBondPortPm15MinIntervalUAS \\
\hline & \\
\hline aGroupPerf1DayIntervalNumber & gBondPortPm1DayIntervalindex \\
\hline aGroupPerf1DayIntervalValid & gBondPortPm1DayIntervalValid \\
\hline aGroupPerf1DayIntervalMoniSecs & gBondPortPm1DayIntervalMoniTime \\
\hline aGroupPerf1DayIntervalES & gBondPortPm1DayIntervalES \\
\hline aGroupperf1 & m1Day Intervo \\
\hline
\end{tabular}




\begin{tabular}{|c|c|}
\hline aGroupPerf1DayIntervalUAS & gBondPortPm1DayIntervalUAS \\
\hline $\begin{array}{l}\text { oLine - Basic Package } \\
\text { (Mandatory) }\end{array}$ & \\
\hline aLineID & ifIndex (IF-MIB) \\
\hline aLineType & iftype (IF-MIB) \\
\hline aLineoperState & ifOperstatus (IF-MIB) \\
\hline aLinestatus & *dsl*Currstatus (*DSL-LINE-MIB) \\
\hline aLineEnd & *dsI*Side (*DSL-LINE-MIB) \\
\hline aLineAdminstate & ifAdminstatus (IF-MIB) \\
\hline aLineRemoteDiscoveryCode & gBondBceConfRemoteDiscoveryCode \\
\hline aLineUpDownEnable & ifLinkUpDownTrapEnable (IF-MIB) \\
\hline nLineUp & LinkUp (IF-MIB) \\
\hline nLineDown & LinkDown (IF-MIB) \\
\hline $\begin{array}{l}\text { oChannel - Basic Package } \\
\text { (Mandatory) }\end{array}$ & \\
\hline aChannelID & ifIndex (IF-MIB) \\
\hline aChannelGroupID & \\
\hline aChannelType & ifType (IF-MIB) \\
\hline aChanneloperstate & ifoperstatus (IF-MIB) \\
\hline aChannelstatus & $\begin{array}{l}\text { *dsl*CurrStatus (*DSL-LINE-MIB), } \\
\text { xdsl2ChStatus*Status } \\
\text { (VDSL2-LINE-MIB) }\end{array}$ \\
\hline
\end{tabular}

Table 2: Mapping of TR-159 Managed Objects 
6. xDSL Multi-Pair Bonding MIB Definitions

The GBOND-MIB module IMPORTS objects from SNMPV2-SMI [RFC2578], SNMPV2-TC [RFC2579], SNMPV2-CONF [RFC2580], SNMP-FRAMEWORK-MIB [RFC3411], IF-MIB [RFC2863], and HC-PerfHist-TC-MIB [RFC3705]. The module has been structured as recommended by [RFC4181].

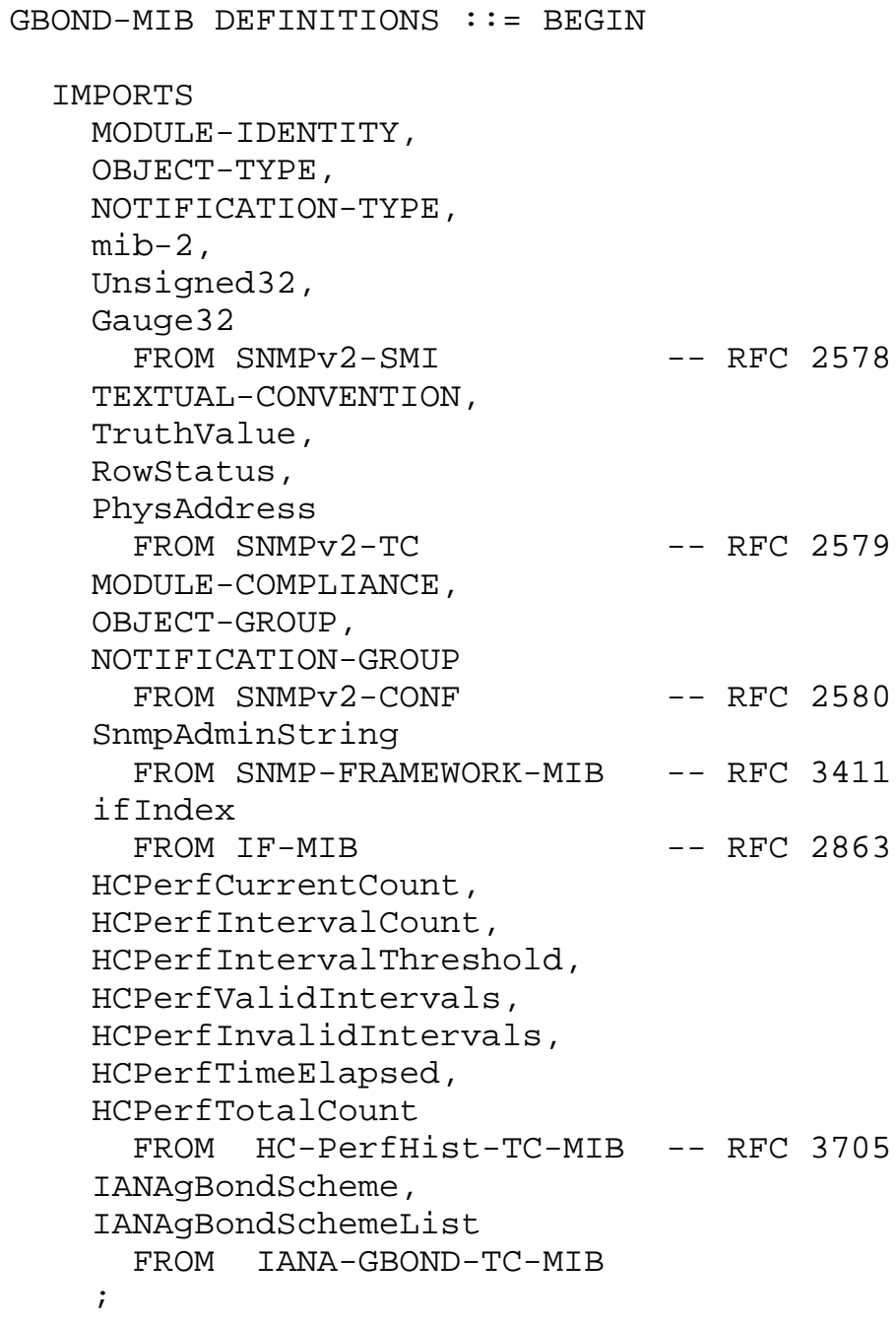




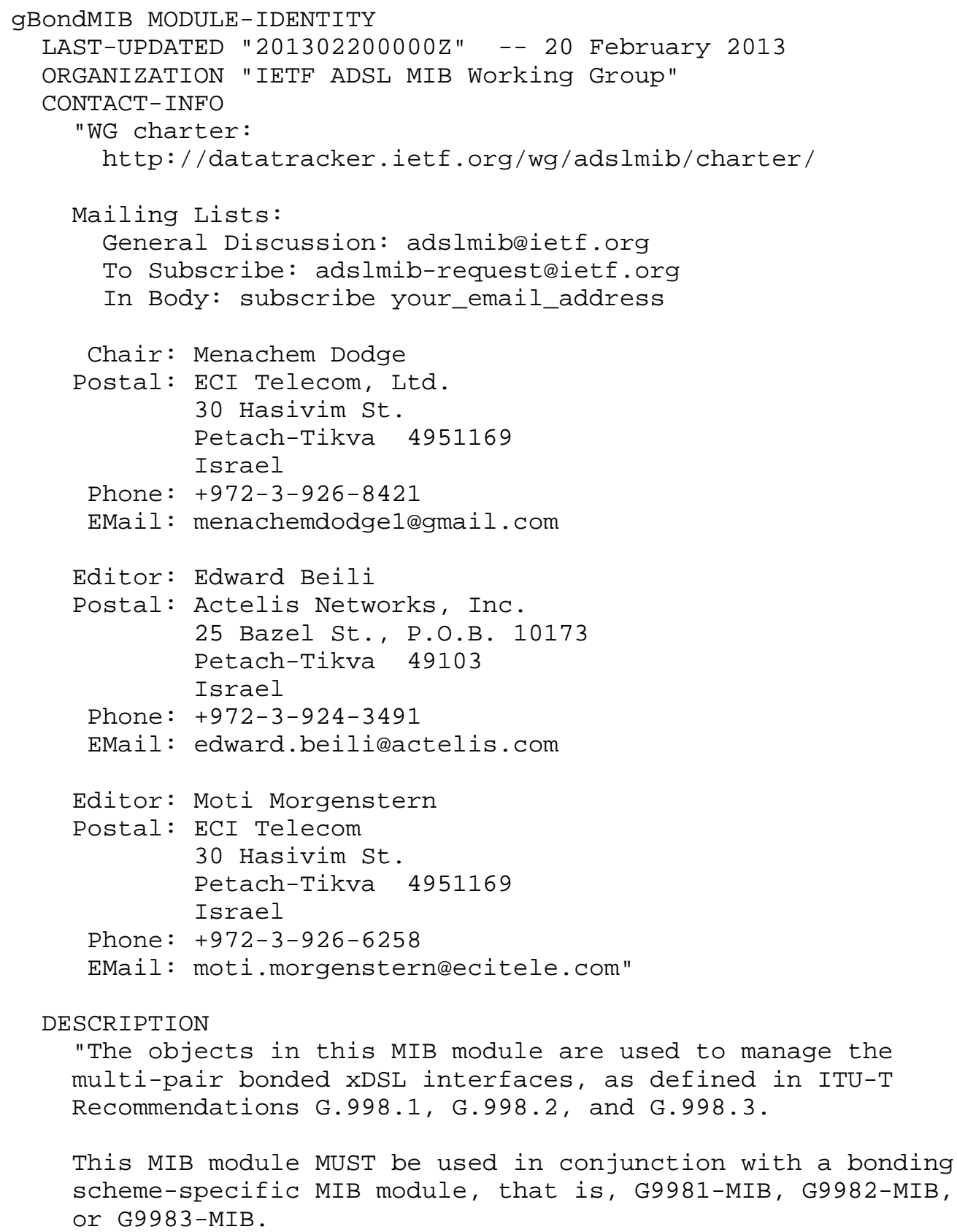


The following references are used throughout this MIB module:

[G.998.1] refers to:

ITU-T Recommendation G.998.1: 'ATM-based multi-pair bonding', January 2005 .

[G.998.2] refers to:

ITU-T Recommendation G.998.2: 'Ethernet-based multi-pair bonding', January 2005 .

[G.998.3] refers to:

ITU-T Recommendation G.998.3: 'Multi-pair bonding using

time-division inverse multiplexing', January 2005.

[TR-159] refers to:

Broadband Forum Technical Report: 'Management Framework for xDSL Bonding', December 2008 .

Naming Conventions:

BCE - Bonding Channel Entity

BTU - Bonding Terminating Unit

BTU-C - Bonding Terminating Unit, CO side

BTU-R - Bonding Terminating Unit, Remote Terminal (CPE) side

$\mathrm{CO}-$ Central Office

CPE - Customer Premises Equipment

GBS - Generic Bonding Sub-layer

PM - Performance Monitoring

SNR - Signal to Noise Ratio

TCA - Threshold Crossing Alert

Copyright (c) 2013 IETF Trust and the persons identified as authors of the code. All rights reserved.

Redistribution and use in source and binary forms, with or without modification, is permitted pursuant to, and subject to the license terms contained in, the Simplified BSD License set forth in Section 4.c of the IETF Trust's Legal Provisions Relating to IETF Documents (http://trustee.ietf.org/license-info)."

REVISION "201302200000Z" -- 20 February 2013

DESCRIPTION "Initial version, published as RFC 6765."

$::=\{\operatorname{mib}-2211\}$

-- Sections of the module

-- Structured as recommended by RFC 4181, Appendix D 


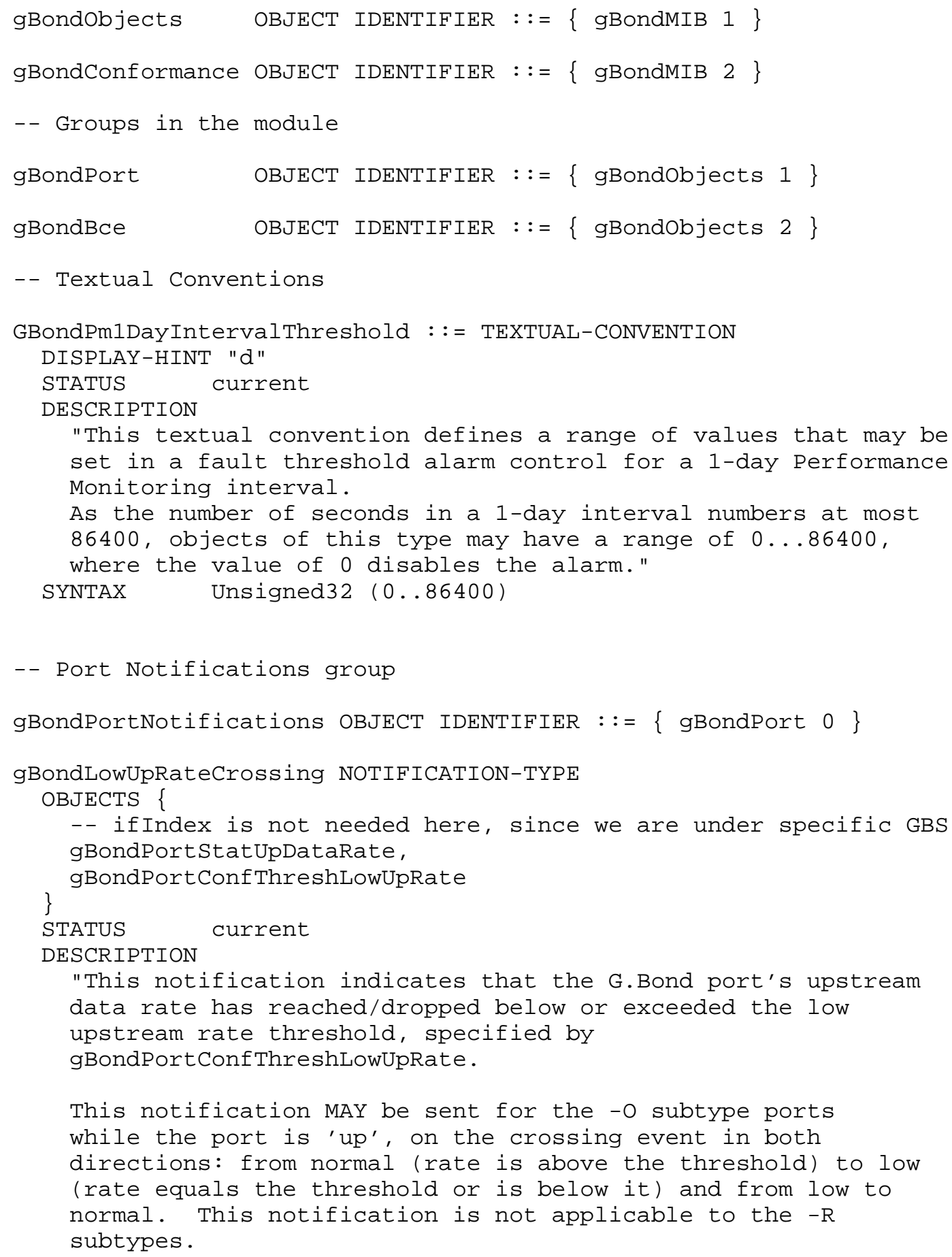


It is RECOMMENDED that a small debouncing period of 2.5 sec, between the detection of the condition and notification, be implemented to prevent simultaneous LinkUp/LinkDown and gBondLowUpRateCrossing notifications from being sent.

The adaptive nature of the G.Bond technology allows the port to adapt itself to the changes in the copper environment; e.g., an impulse noise, alien crosstalk, or a micro-interruption may temporarily drop one or more BCEs in the aggregation group, causing a rate degradation of the aggregated G.Bond link. The dropped BCEs would then try to re-initialize, possibly at a lower rate than before, adjusting the rate to provide the required target SNR margin.

Generation of this notification is controlled by the gBondPortConfLowRateCrossingEnable object.

This object maps to the TR-159 notification

nGroupLowUpRateCrossing."

REFERENCE

"[TR-159], Section 5.5.1.24"

$::=\{$ gBondPortNotifications 1$\}$

gBondLowDnRateCrossing NOTIFICATION-TYPE

OBJECTS \{

-- ifIndex is not needed here, since we are under specific GBS gBondPortstatDnDataRate, \} gBondPortConfThreshLowDnRate

STATUS current

DESCRIPTION

"This notification indicates that the G.Bond port's downstream data rate has reached/dropped below or exceeded the low downstream rate threshold, specified by gBondPortConfthreshLowDnRate.

This notification MAY be sent for the -O subtype ports while the port is 'up', on the crossing event in both directions: from normal (rate is above the threshold) to low (rate equals the threshold or is below it) and from low to normal. This notification is not applicable to the $-\mathrm{R}$ subtypes.

It is RECOMMENDED that a small debouncing period of 2.5 sec, between the detection of the condition and notification, be implemented to prevent simultaneous LinkUp/LinkDown and gBondLowDnRateCrossing notifications from being sent. 
The adaptive nature of the G.Bond technology allows the port to adapt itself to the changes in the copper environment; e.g., an impulse noise, alien crosstalk, or a micro-interruption may temporarily drop one or more BCEs in the aggregation group, causing a rate degradation of the aggregated G.Bond link. The dropped BCEs would then try to re-initialize, possibly at a lower rate than before, adjusting the rate to provide the required target SNR margin.

Generation of this notification is controlled by the gBondPortConfLowRateCrossingEnable object.

This object maps to the TR-159 notification

nGroupLowDownRateCrossing."

REFERENCE

"[TR-159], Section 5.5.1.25"

$::=\{$ gBondPortNotifications 2$\}$

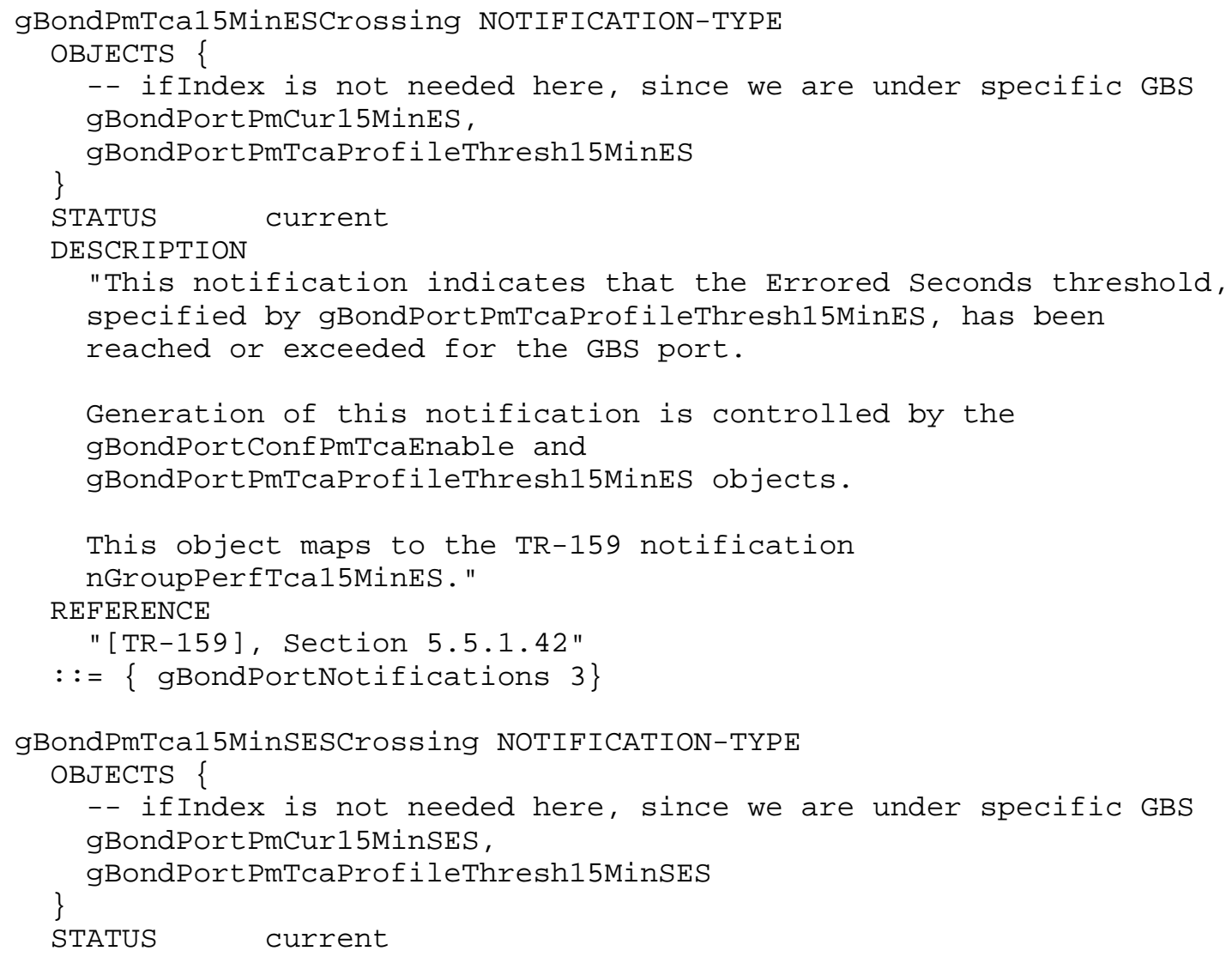




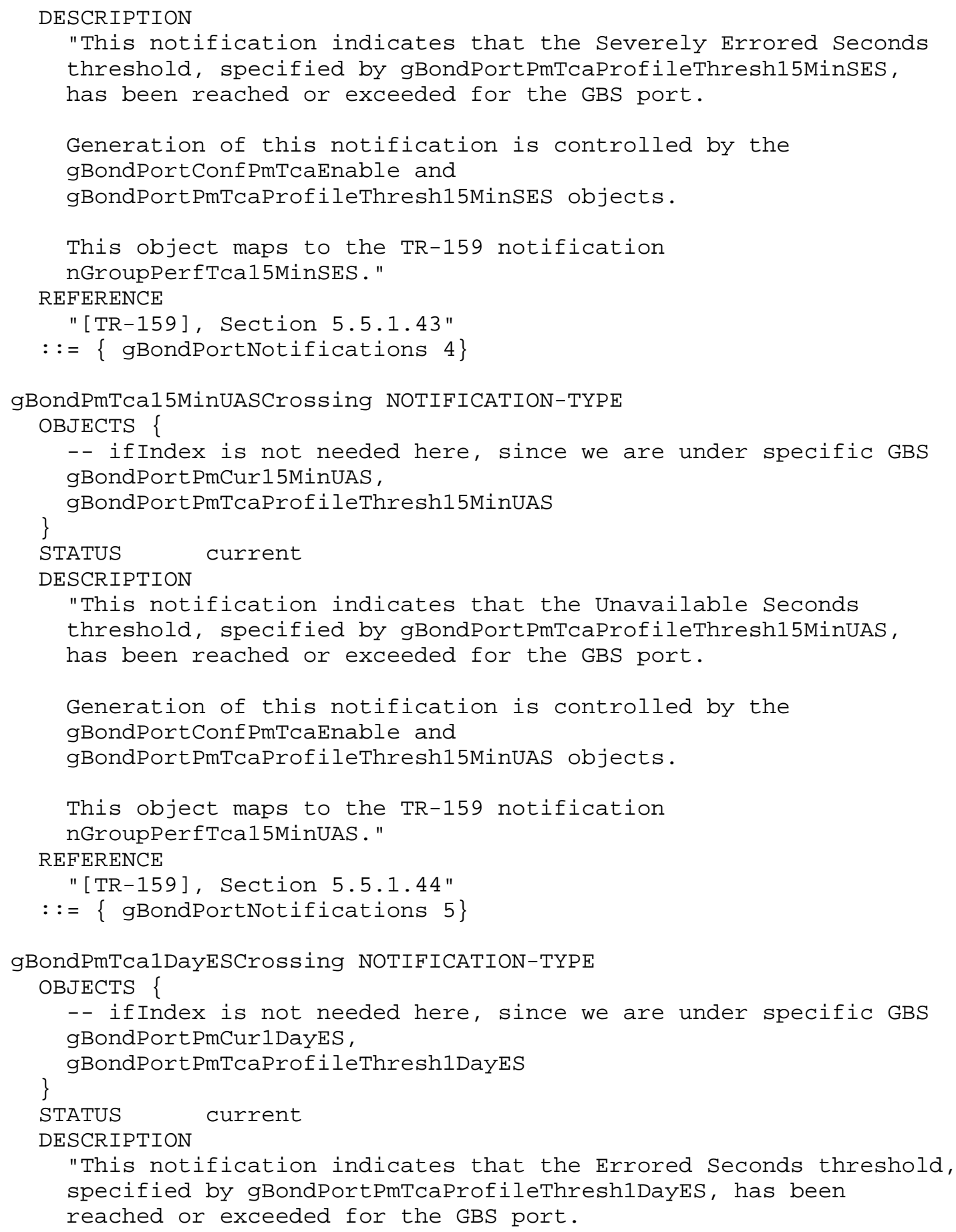




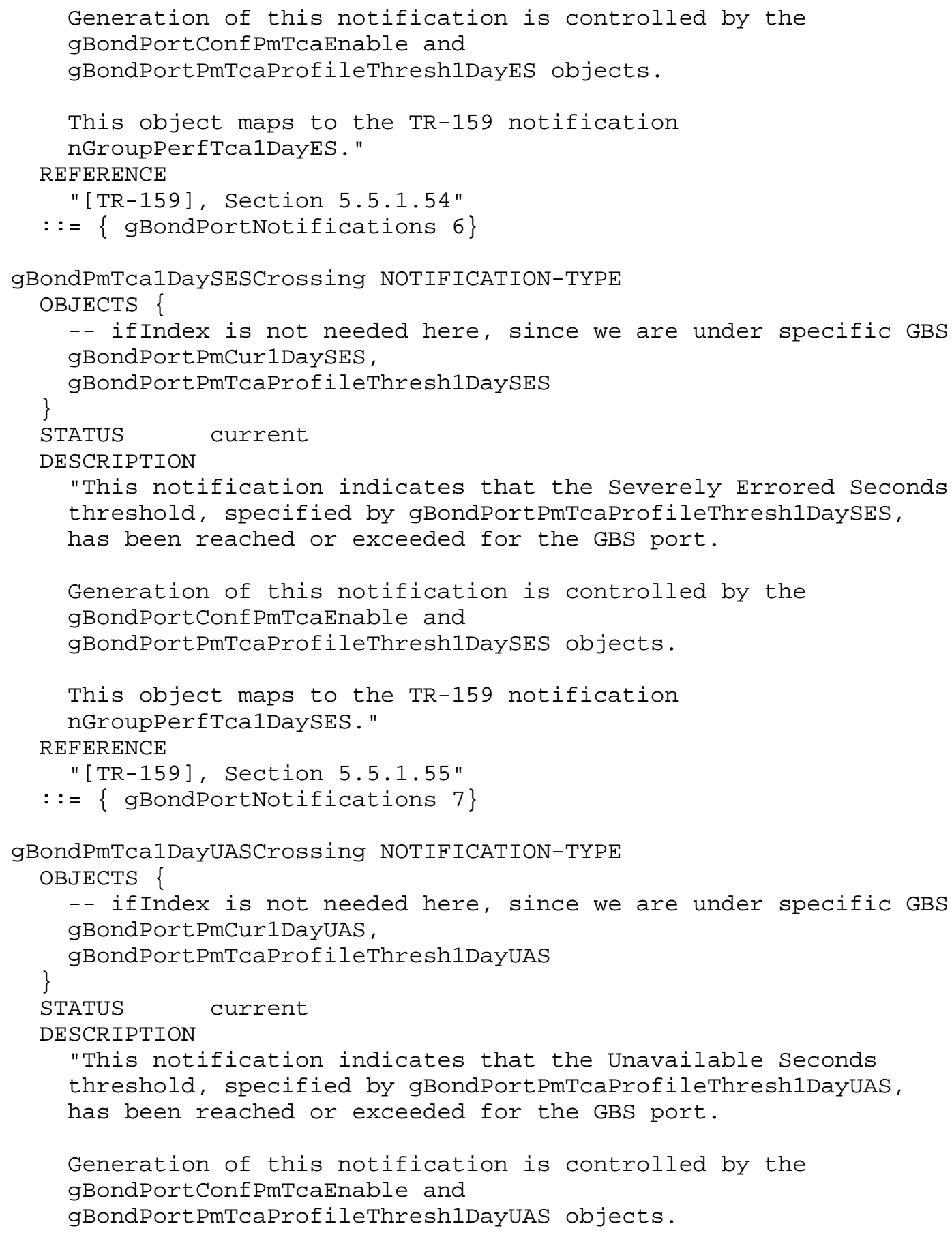




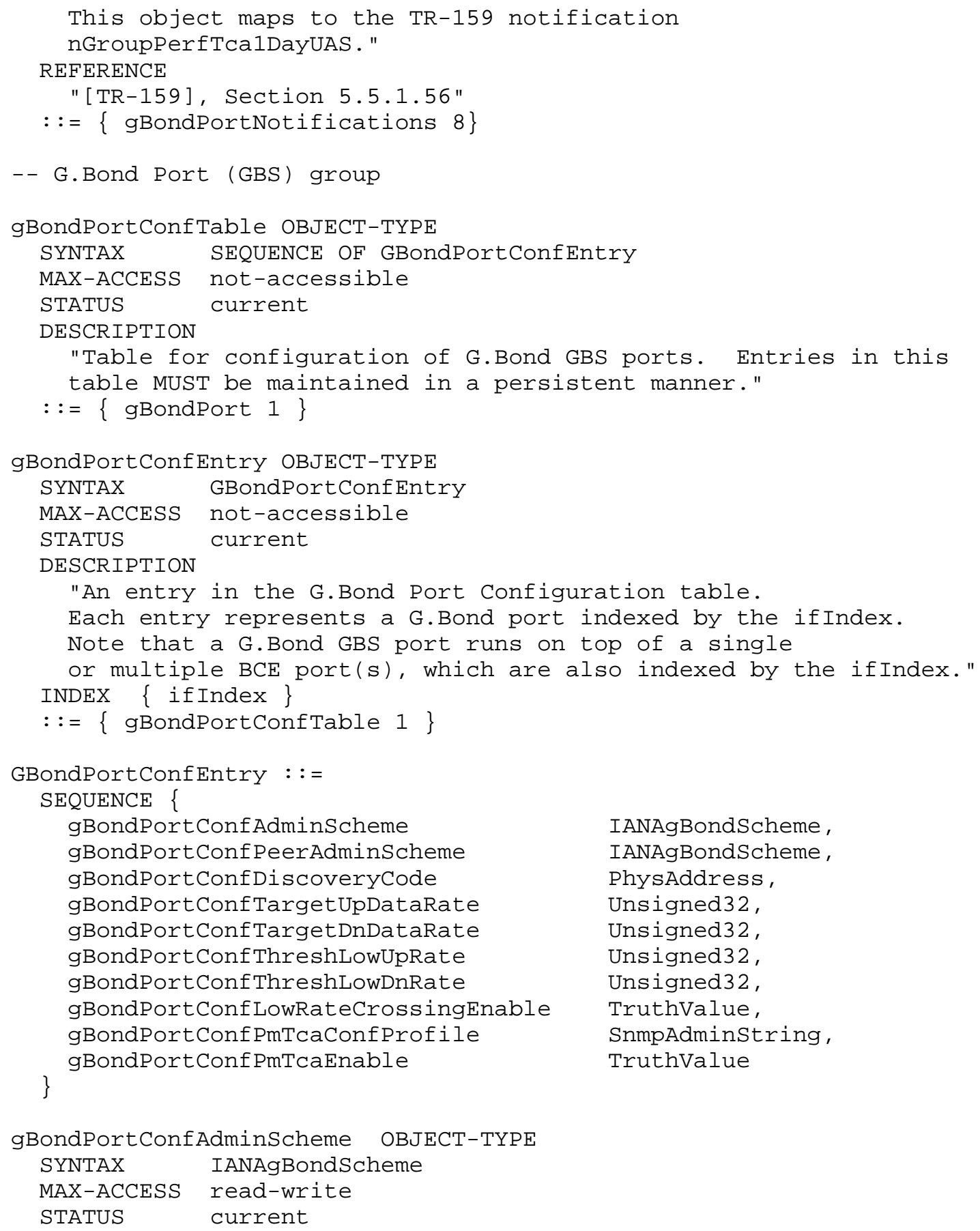




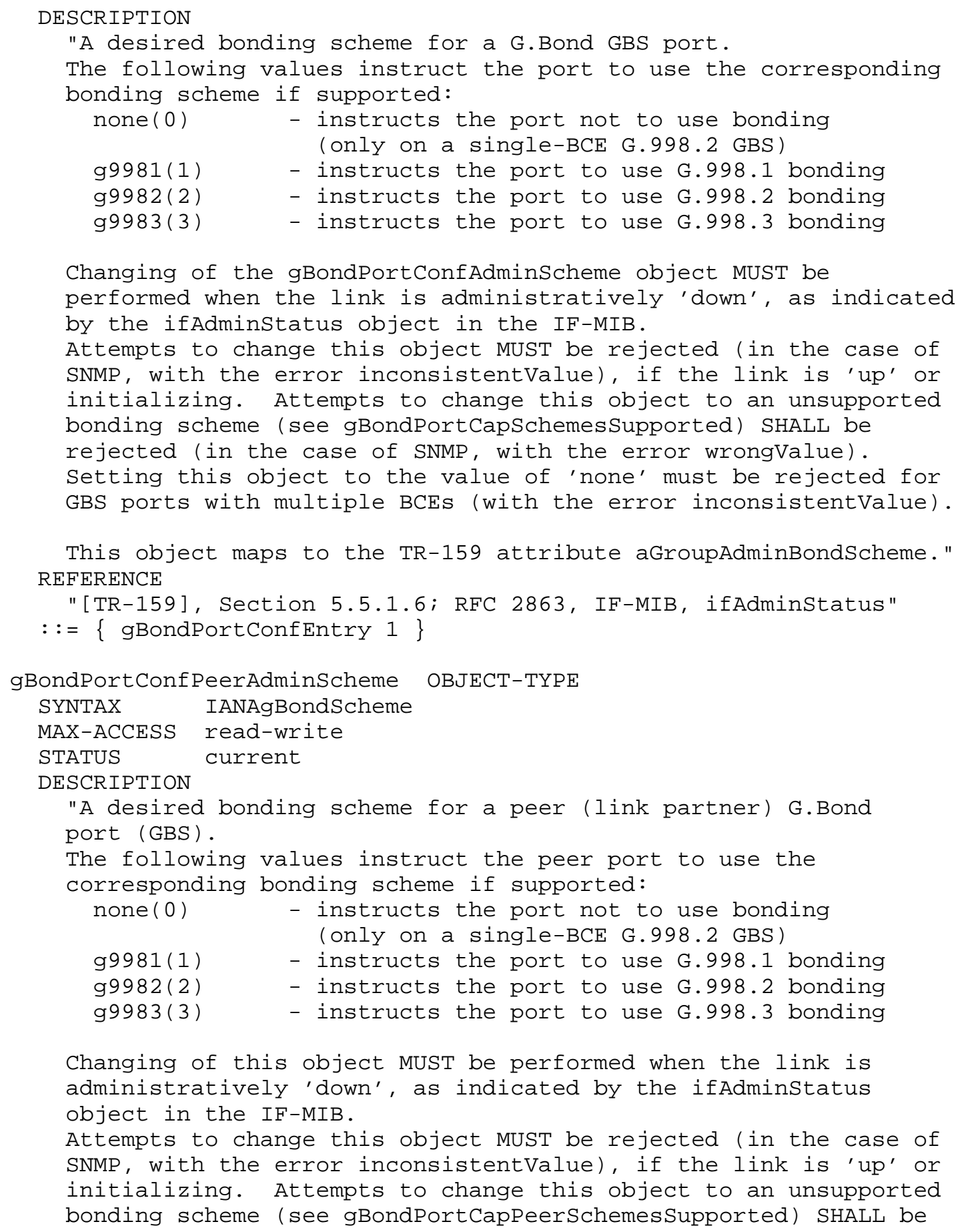

Changing of this object MUST be performed when the link is administratively 'down', as indicated by the ifAdminstatus object in the IF-MIB.

Attempts to change this object MUST be rejected (in the case of SNMP, with the error inconsistentValue), if the link is 'up' or initializing. Attempts to change this object to an unsupported bonding scheme (see gBondPortCapPeerSchemesSupported) SHALL be 


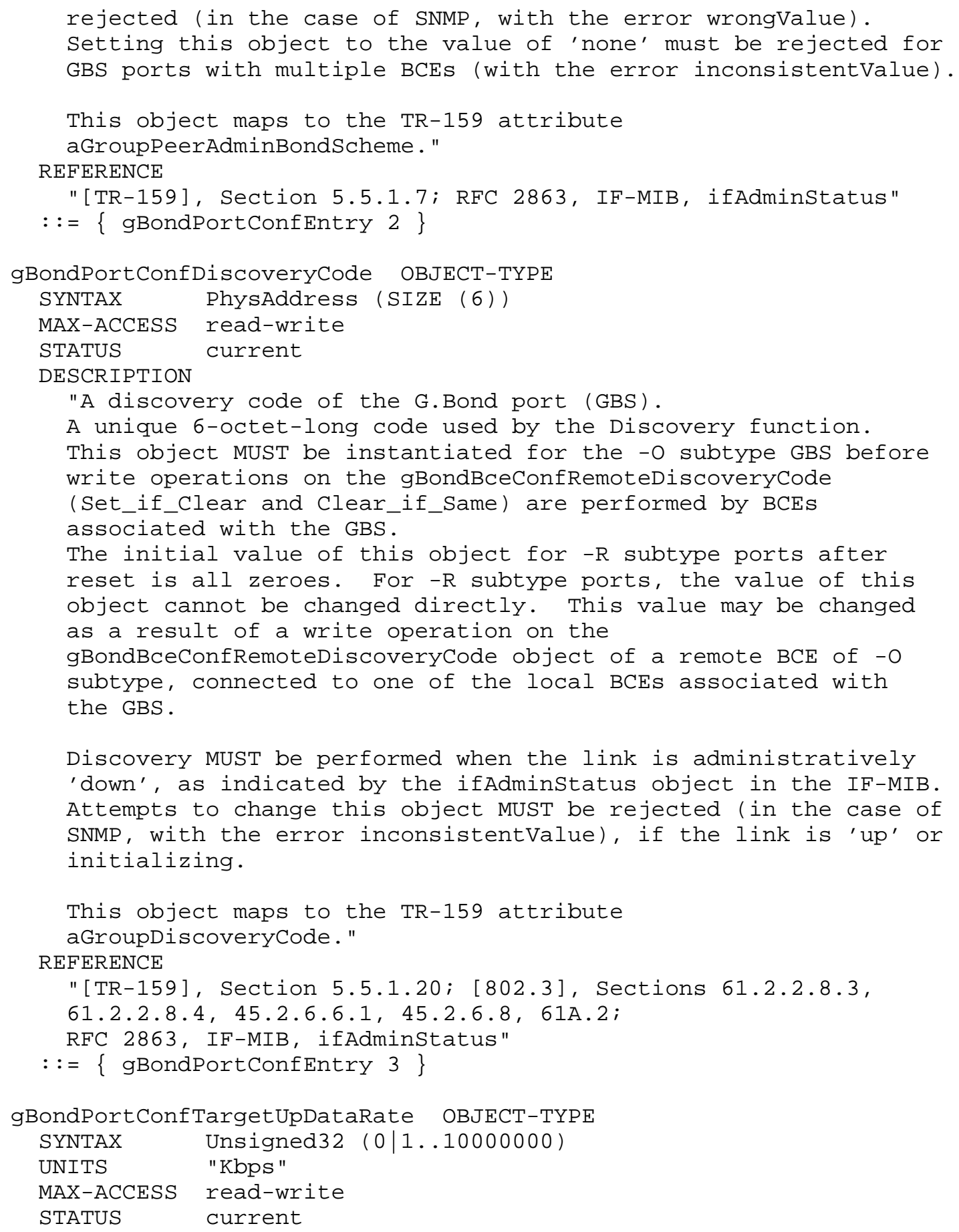




\section{DESCRIPTION}

"A desired G.Bond port data rate in the upstream direction, in Kbps, to be achieved during initialization, under restrictions placed upon the member BCEs by their respective configuration settings.

This object represents a sum of individual BCE upstream data rates, modified to compensate for fragmentation and encapsulation overhead (e.g., for an Ethernet service, the target data rate of 10 Mbps SHALL allow lossless transmission of full-duplex 10-Mbps Ethernet frame stream with minimal inter-frame gap). Note that the target upstream data rate may not be achieved during initialization (e.g., due to unavailability of required BCES) or the initial bandwidth could deteriorate, so that the actual upstream data rate (gBondPortstatUpDataRate) could be less than gBondPortConfTargetUpDataRate.

The value is limited above by 10 Gbps, to accommodate very high speed bonded XDSL interfaces (e.g., 32 x 100 Mbps).

The value between 1 and 10000000 indicates that the total upstream data rate of the G.Bond port after initialization SHALL be equal to the target data rate or less, if the target upstream data rate cannot be achieved under the restrictions configured for BCEs. In cases where the copper environment allows a higher upstream data rate to be achieved than that specified by this object, the excess capability SHALL be either converted to an additional SNR margin or reclaimed by minimizing transmit power.

The value of 0 means that the target data rate is not fixed and SHALL be set to the maximum attainable rate during initialization (best effort), under specified spectral restrictions and with a desired SNR margin per BCE.

This object is read-write for the -O subtype G.Bond ports. It is irrelevant for the - $\mathrm{R}$ subtypes -- attempts to read or change this object for such ports MUST be rejected (in the case of SNMP, with the error inconsistentvalue).

Changing of the target upstream data rate MUST be performed when the link is administratively 'down', as indicated by the ifAdminstatus object in the IF-MIB.

Attempts to change this object MUST be rejected (in the case of SNMP, with the error inconsistentValue), if the link is 'up' or initializing.

This object maps to the TR-159 attribute aGroupTargetUpRate." 


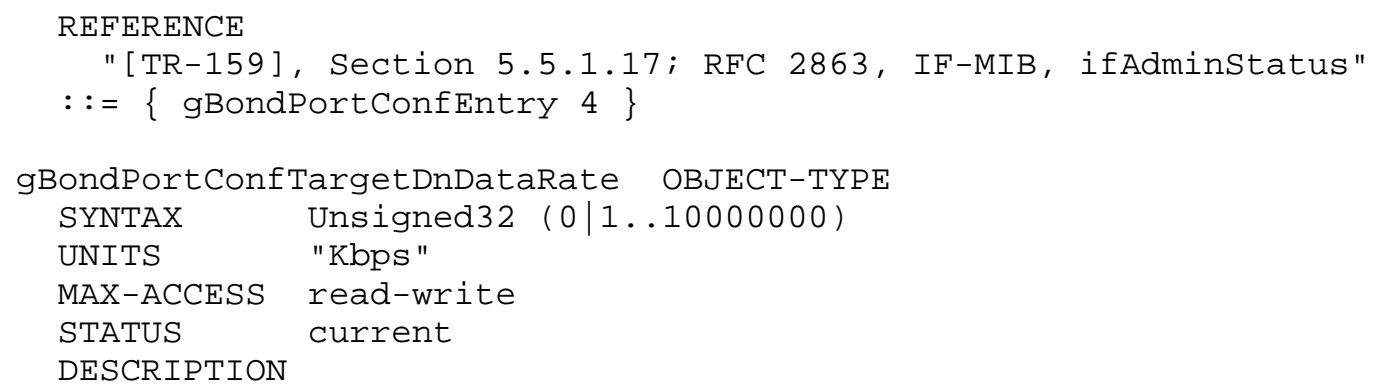

"A desired G.Bond port data rate in the downstream direction, in Kbps, to be achieved during initialization, under restrictions placed upon the member BCEs by their respective configuration settings.

This object represents a sum of individual BCE downstream data rates, modified to compensate for fragmentation and encapsulation overhead (e.g., for an Ethernet service, the target data rate of 10 Mbps SHALL allow lossless transmission of full-duplex 10-Mbps Ethernet frame stream with minimal inter-frame gap). Note that the target downstream data rate may not be achieved during initialization (e.g., due to unavailability of required BCES) or the initial bandwidth could deteriorate, so that the actual downstream data rate (gBondPortstatDnDataRate) could be less than gBondPortConfTargetDnDataRate.

The value is limited above by 10 Gbps, to accommodate very high speed bonded XDSL interfaces (e.g., 32 x 100 Mbps).

The value between 1 and 10000000 indicates that the total downstream data rate of the G.Bond port after initialization SHALL be equal to the target data rate or less, if the target downstream data rate cannot be achieved under the restrictions configured for BCEs. In cases where the copper environment allows a higher downstream data rate to be achieved than that specified by this object, the excess capability SHALL be either converted to an additional SNR margin or reclaimed by minimizing transmit power.

The value of 0 means that the target data rate is not fixed and SHALL be set to the maximum attainable rate during initialization (best effort), under specified spectral restrictions and with a desired SNR margin per BCE.

This object is read-write for the -O subtype G.Bond ports. It is irrelevant for the -R subtypes -- attempts to read or change this object for such ports MUST be rejected (in the case of SNMP, with the error inconsistentValue). 


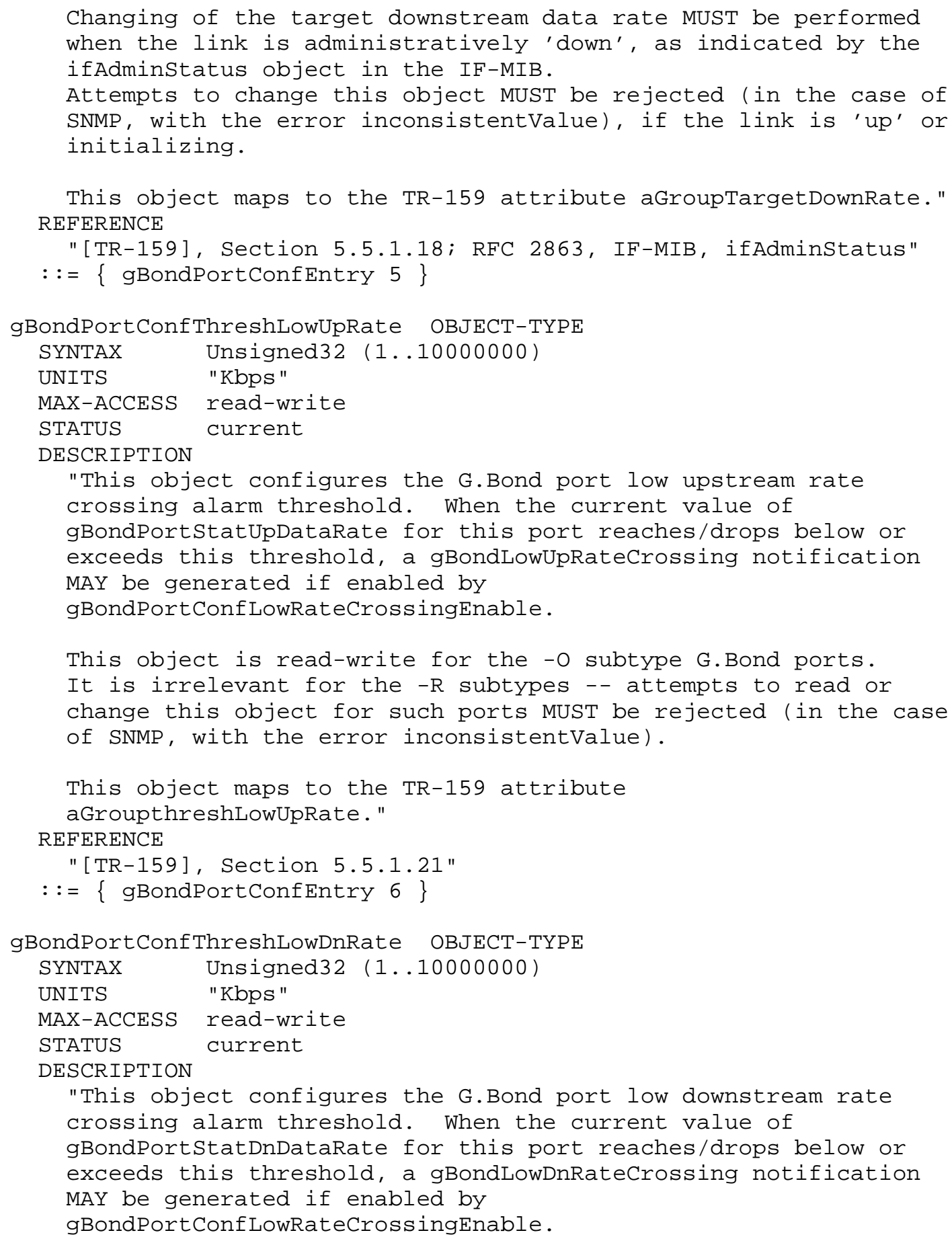




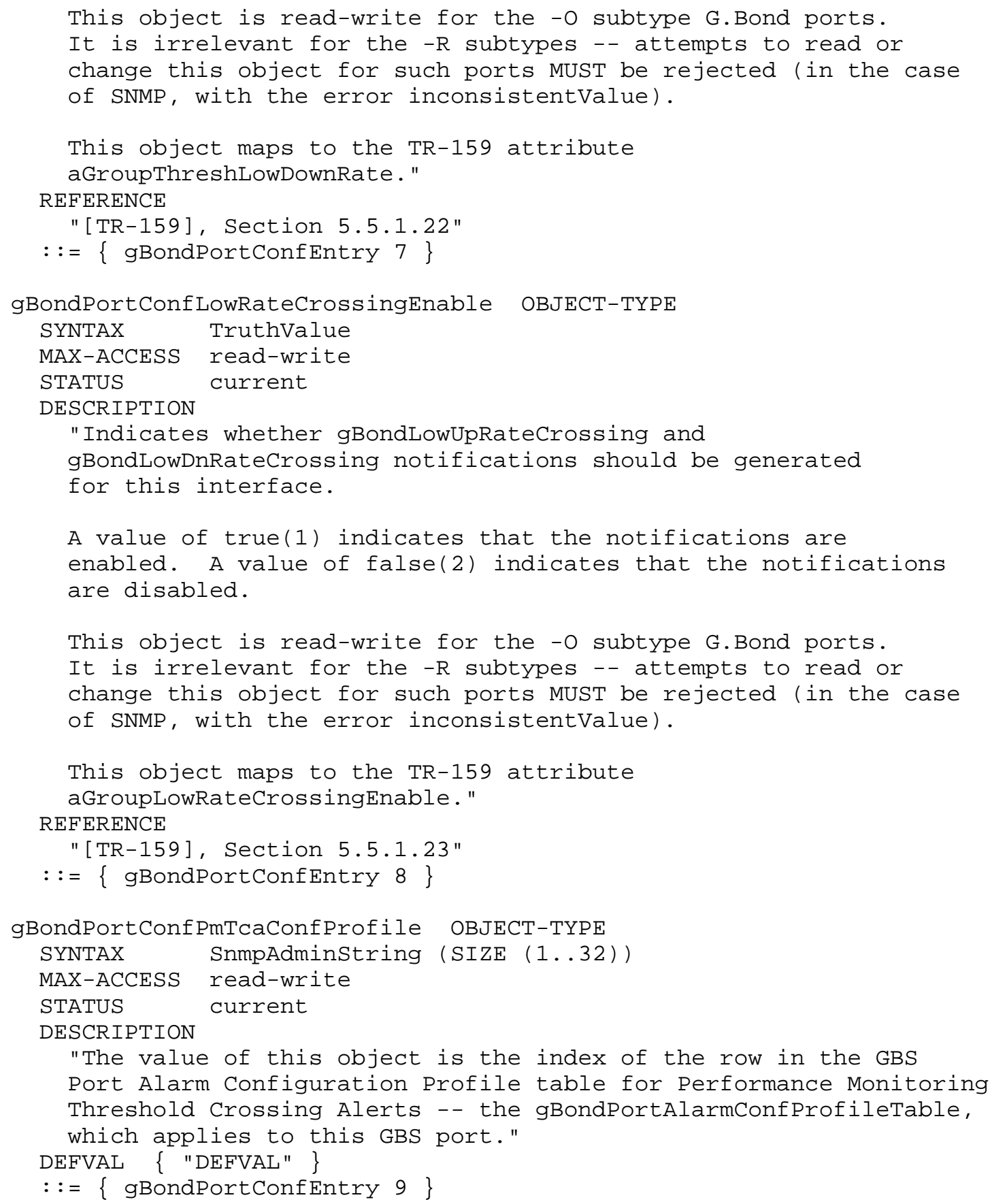




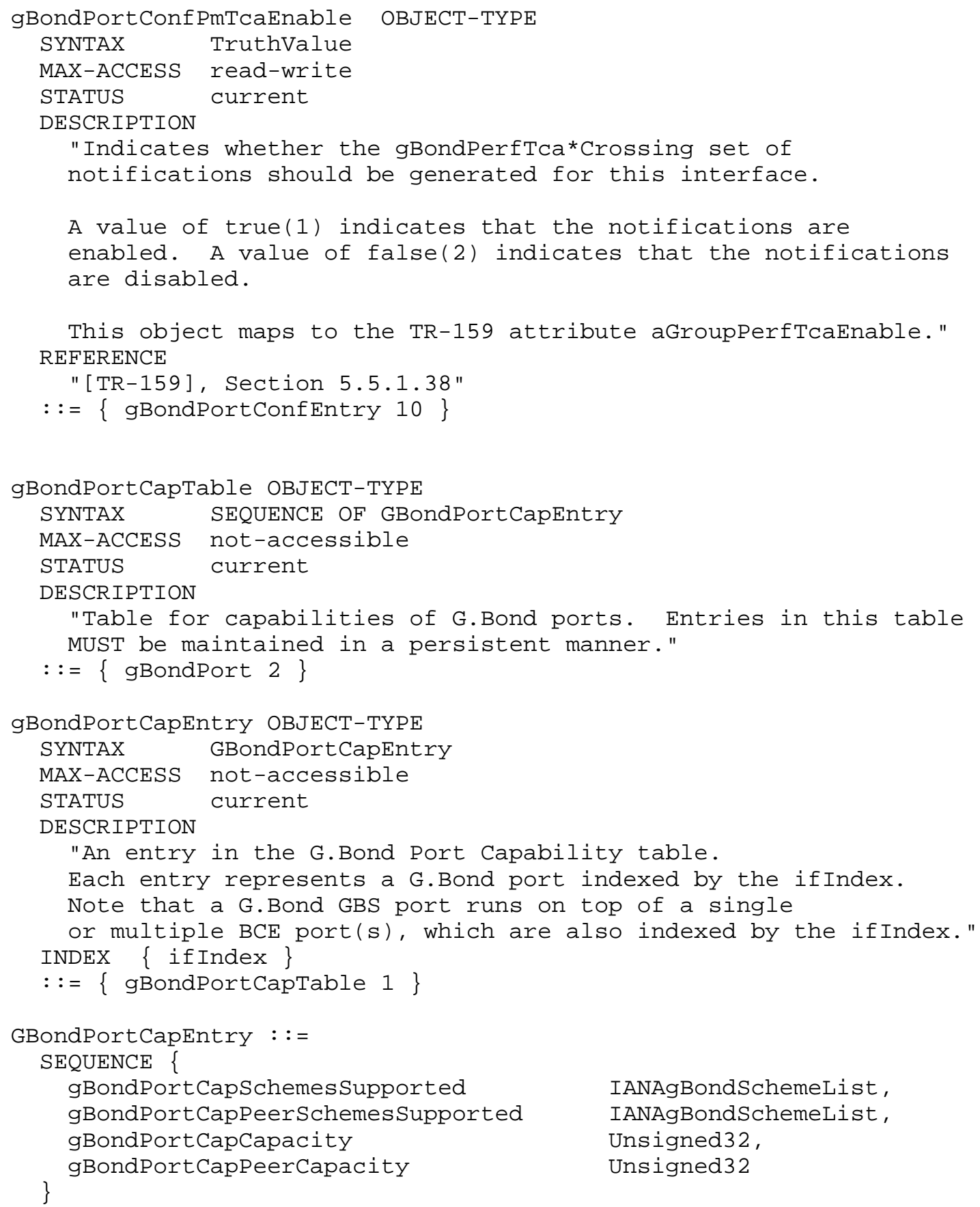




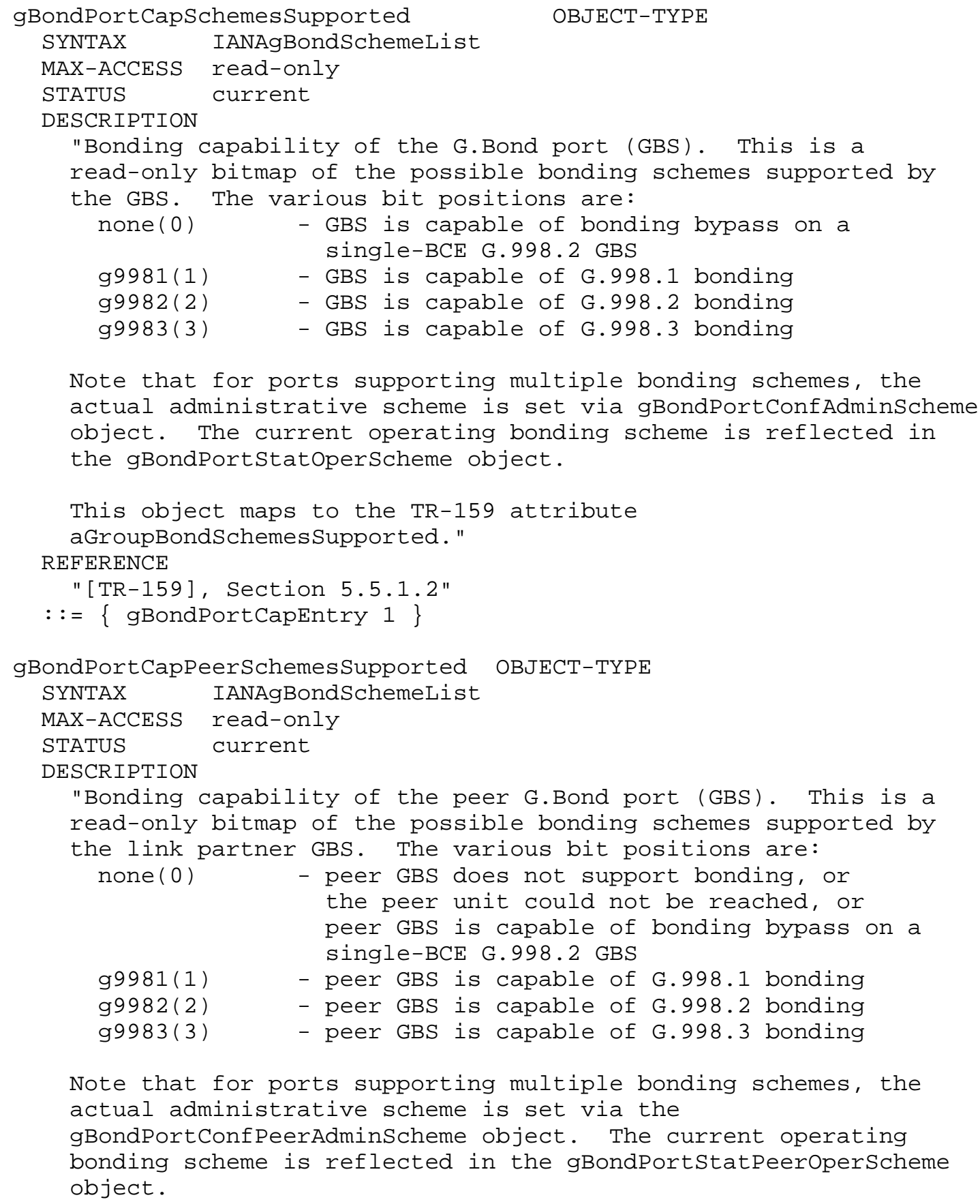

Note that for ports supporting multiple bonding schemes, the actual administrative scheme is set via gBondPortConfAdminscheme object. The current operating bonding scheme is reflected in the gBondPortstatoperScheme object.

This object maps to the TR-159 attribute aGroupBondSchemes Supported." REFERENCE

"[TR-159], Section 5.5.1.2"

$::=\{$ gBondPortCapentry 1$\}$

\section{gBondPortCapPeerSchemesSupported OBJECT-TYPE}

SYNTAX IANAgBondSchemeList

MAX-ACCESS read-only

STATUS current

DESCRIPTION

"Bonding capability of the peer G.Bond port (GBS). This is a

read-only bitmap of the possible bonding schemes supported by

the link partner GBS. The various bit positions are:

none(0) - peer GBS does not support bonding, or the peer unit could not be reached, or peer GBS is capable of bonding bypass on a single-BCE G.998.2 GBS

g9981(1) - peer GBS is capable of G.998.1 bonding

g9982(2) - peer GBS is capable of G.998.2 bonding

g9983(3) - peer GBS is capable of G.998.3 bonding

Note that for ports supporting multiple bonding schemes, the actual administrative scheme is set via the gBondPortConfPeerAdminScheme object. The current operating bonding scheme is reflected in the gBondPortstatPeeroperscheme object. 


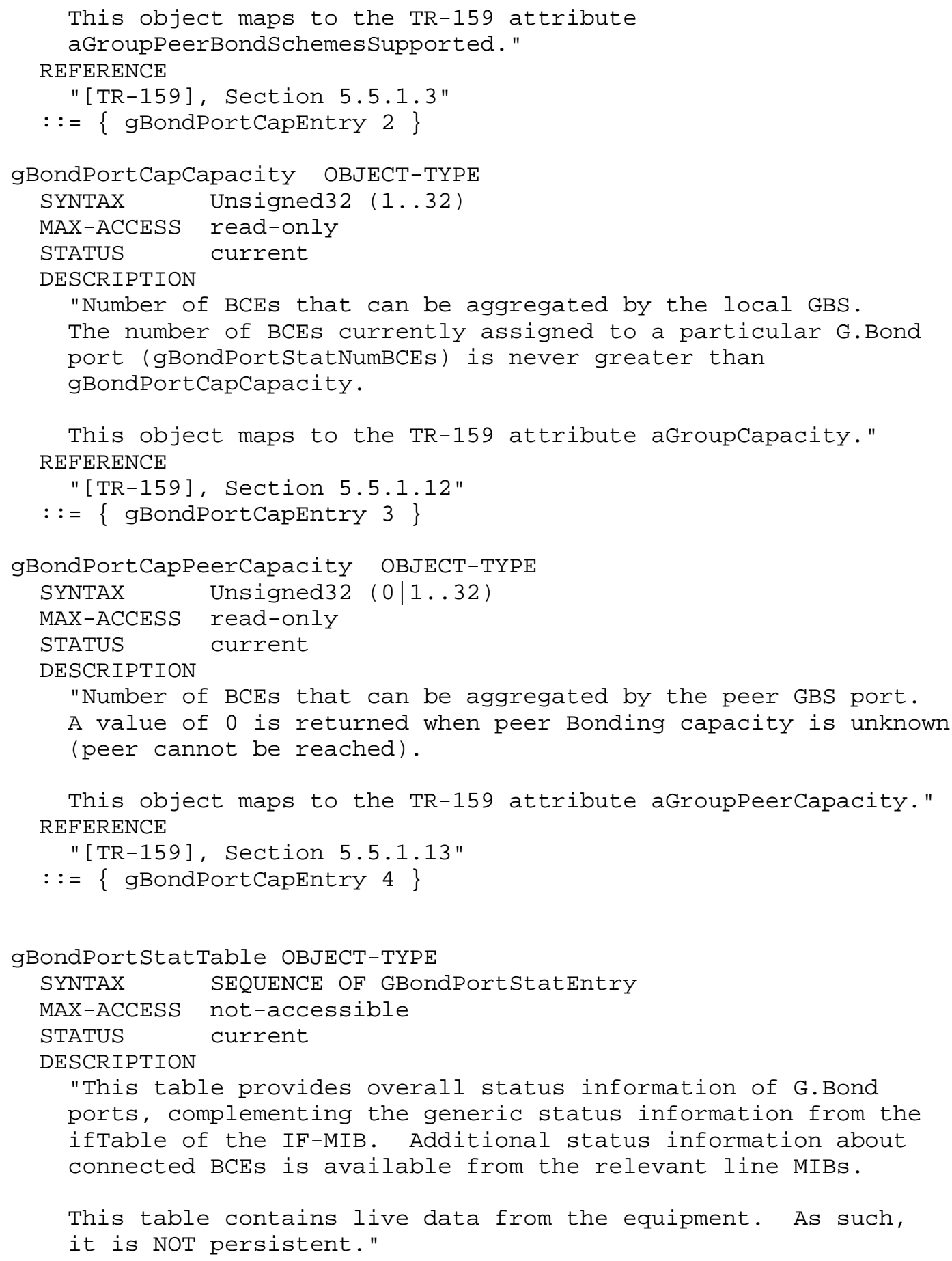




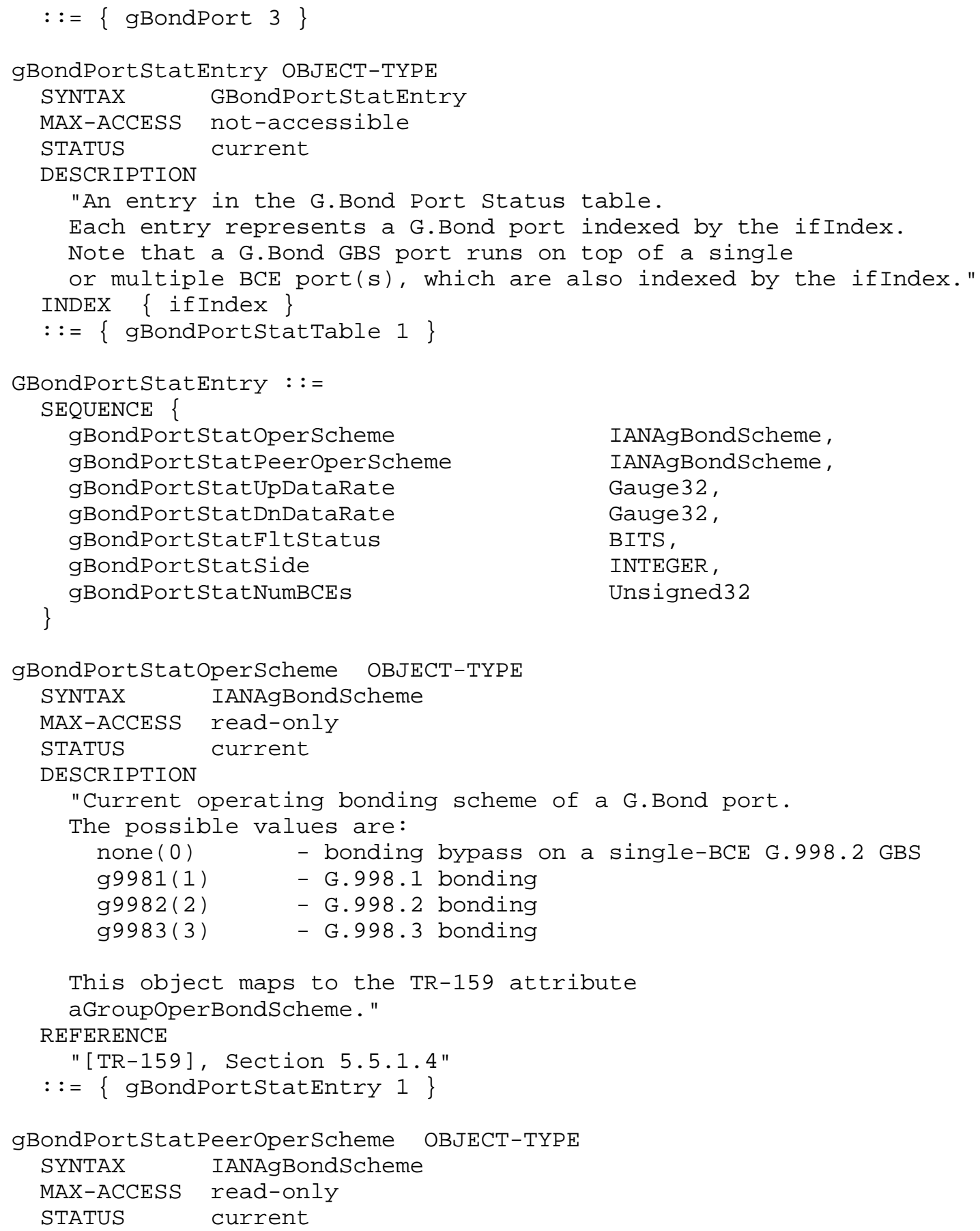




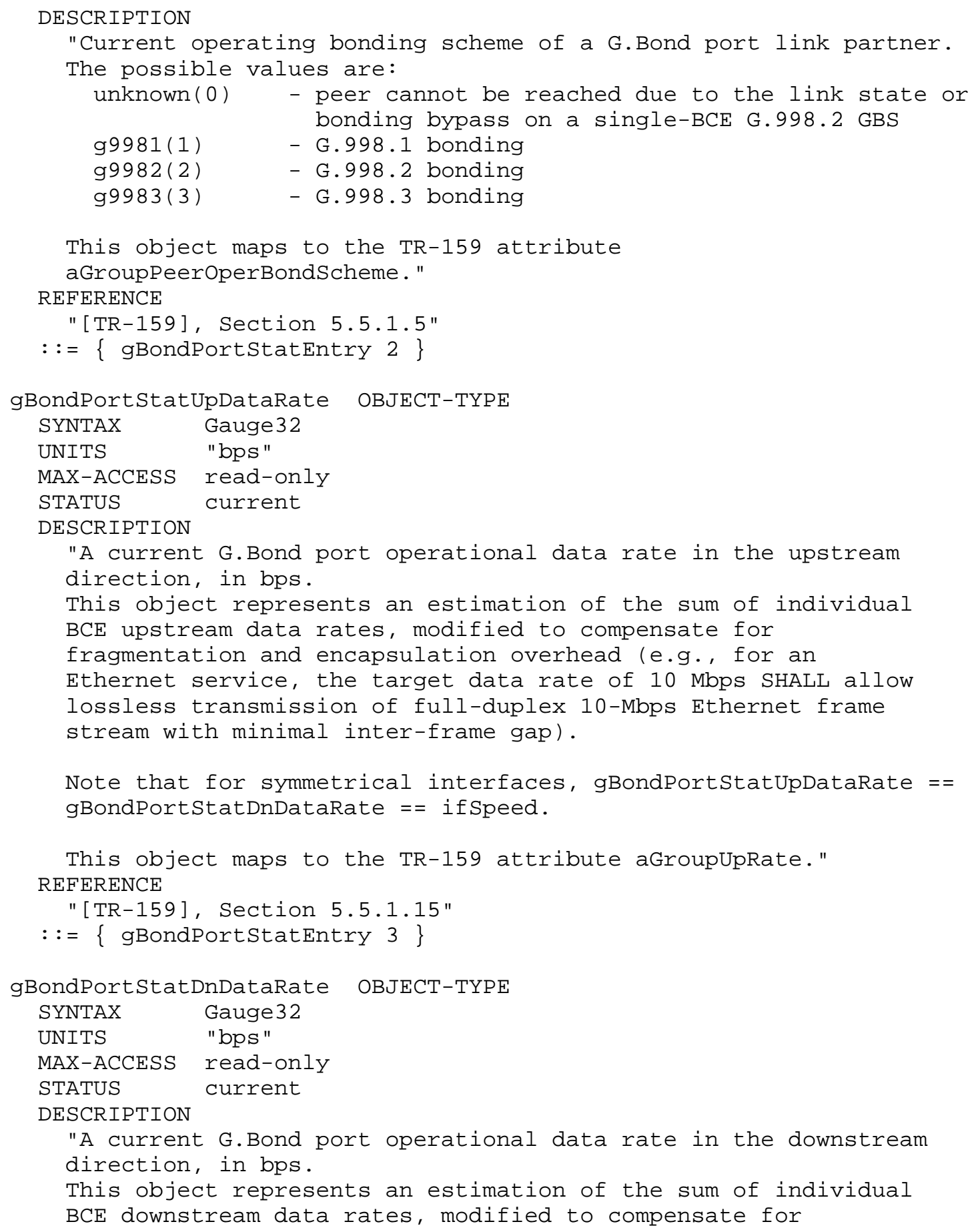




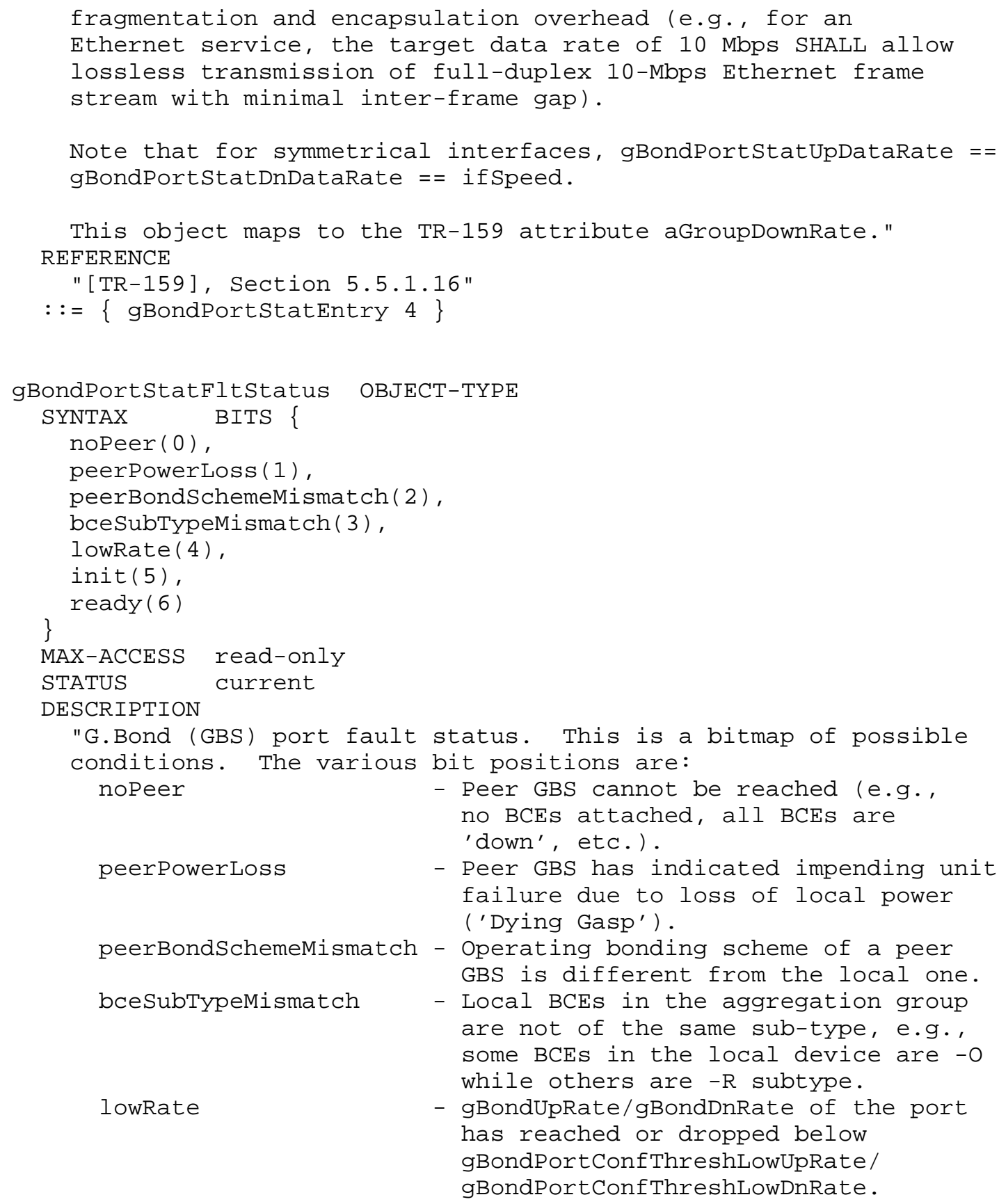


init

ready
- The link is initializing, as a result of ifAdminstatus being set to 'up' for a particular BCE or a GBS to which the BCE is connected.

- At least one BCE in the aggregation group is detecting handshake tones.

This object is intended to supplement the ifoperstatus object in the IF-MIB.

This object maps to the TR-159 attribute aGroupstatus." REFERENCE

"[TR-159], Section 5.5.1.9; RFC 2863, IF-MIB, ifoperStatus" $::=\{$ gBondPortstatentry 5$\}$

gBondPortstatside OBJECT-TYPE

SYNTAX INTEGER \{

subscriber (1),

office (2),

unknown (3)

\}

MAX-ACCESS read-only

STATUS current

DESCRIPTION

"G.Bond port mode of operation (subtype).

The value of 'subscriber' indicates that the port is

designated as ' $-\mathrm{R}^{\prime}$ subtype (all BCEs assigned to this port are of subtype ' $-\mathrm{R}^{\prime}$ ) .

The value of 'office' indicates that the port is designated

as '-O' subtype (all BCEs assigned to this port are of

subtype '-O').

The value of 'unknown' indicates that the port has no assigned

BCEs yet or that the assigned BCEs are not of the same side

(subTypeBCEMismatch).

This object maps to the TR-159 attribute aGroupEnd." REFERENCE

"[TR-159], Section 5.5.1.11"

$::=\{$ gBondPortstatEntry 6$\}$

gBondPortstatNumBCES OBJECT-TYPE

SYNTAX Unsigned32 (0.32)

MAX-ACCESS read-only

STATUS current

DESCRIPTION

"Number of BCEs that are currently aggregated by the local GBS

(assigned to the G.Bond port using the ifstackTable).

This number is never greater than gBondPortCapcapacity. 


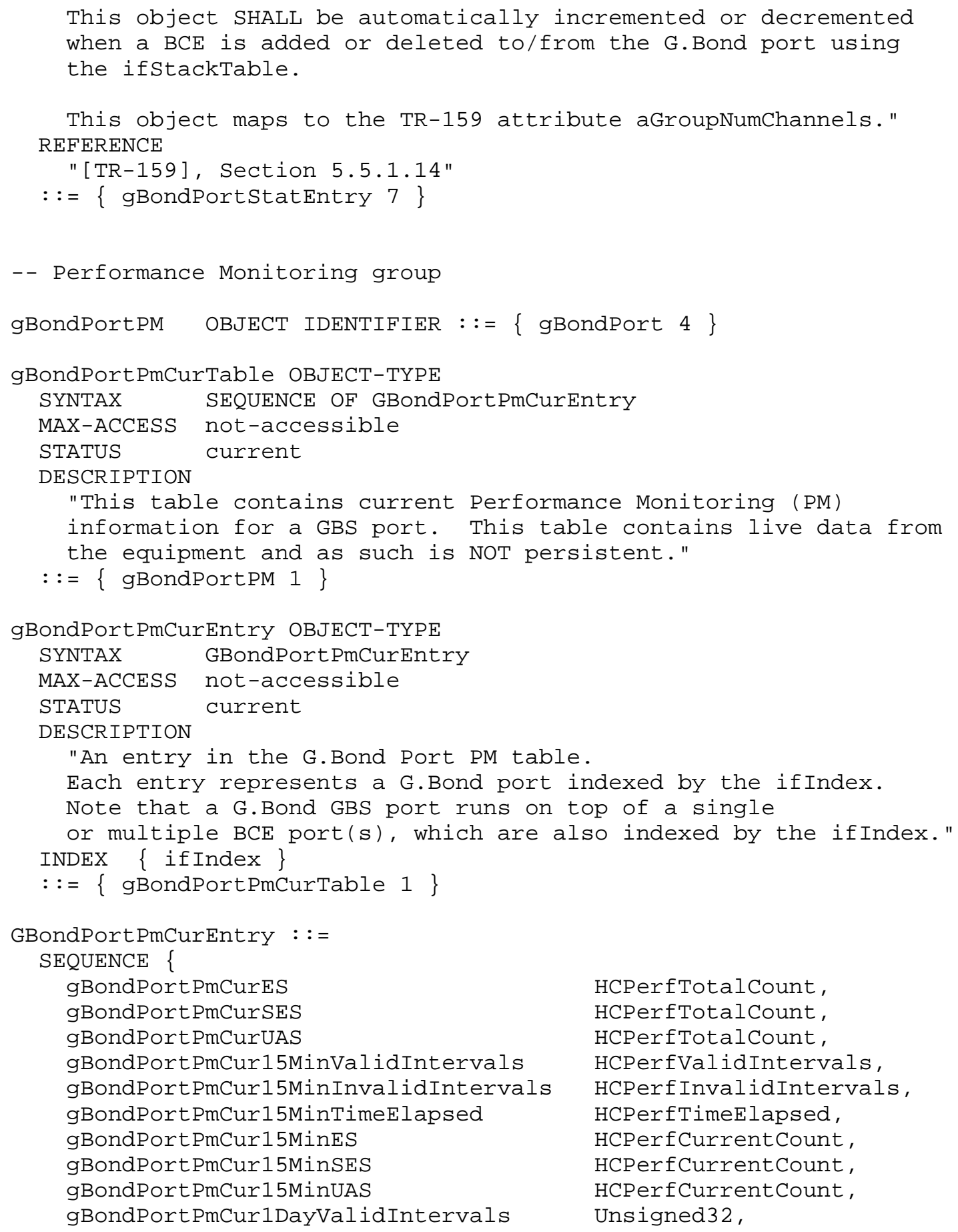




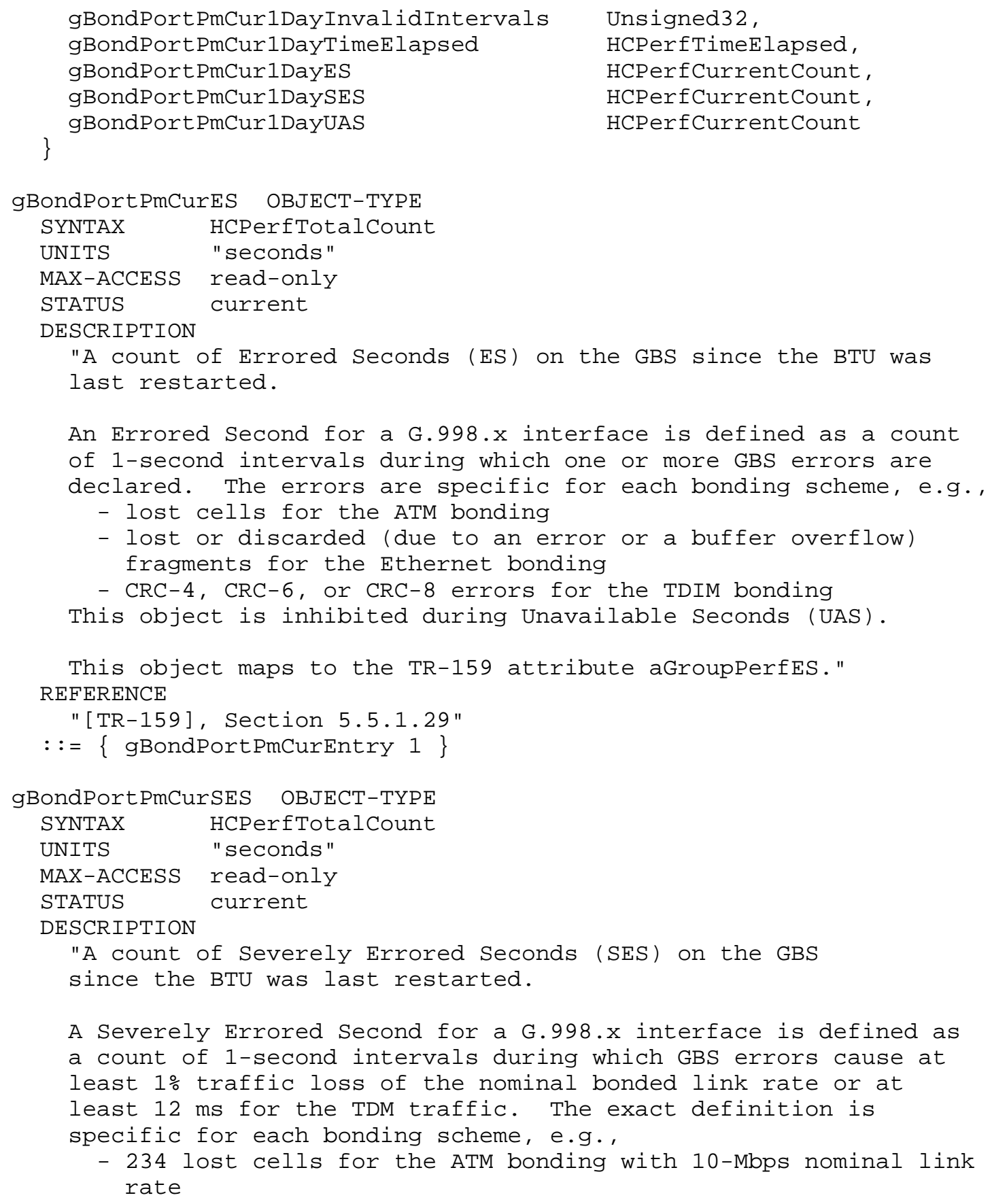


- 60 lost/discarded fragments for the Ethernet bonding with 10-Mbps nominal link rate and fixed 192-byte-long fragment size

- 6 or more CRC-4 errors, one or more CRC- 6 errors, or one or more CRC-8 errors for the TDM bonding

This object is inhibited during Unavailable Seconds (UAS).

This object maps to the TR-159 attribute aGroupPerfSES." REFERENCE

"[TR-159], Section 5.5.1.30"

$::=\{$ gBondPortPmCurEntry 2$\}$

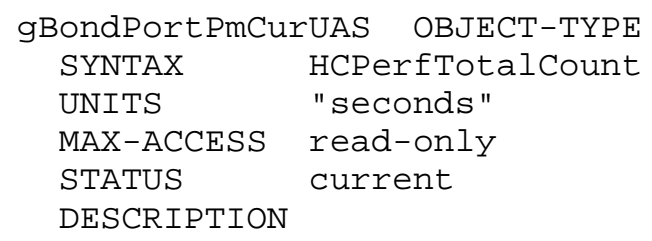

"A count of Unavailable Seconds (UAS) on the GBS since the BTU was last restarted.

An Unavailable second for a G.998.x interface is defined as a count of 1 -second intervals during which the bonded link is unavailable. The G.998.x link becomes unavailable at the onset of 10 contiguous SESS. The 10 SESs are included in the unavailable time. Once unavailable, the G.998.x line becomes available at the onset of 10 contiguous seconds with no SESs. The 10 seconds with no SESs are excluded from the unavailable time.

This object maps to the TR-159 attribute aGroupPerfUAS." REFERENCE

"[TR-159], Section 5.5.1.31"

$::=\{$ gBondPortPmCurEntry 3$\}$

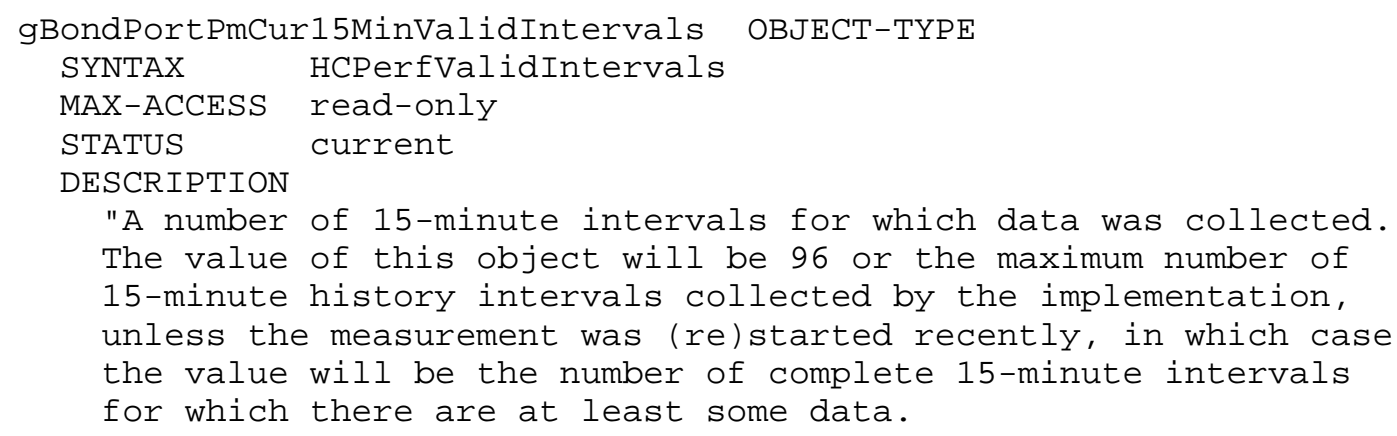

"A number of 15-minute intervals for which data was collected. The value of this object will be 96 or the maximum number of 15-minute history intervals collected by the implementation, unless the measurement was (re) started recently, in which case the value will be the number of complete 15-minute intervals for which there are at least some data. 


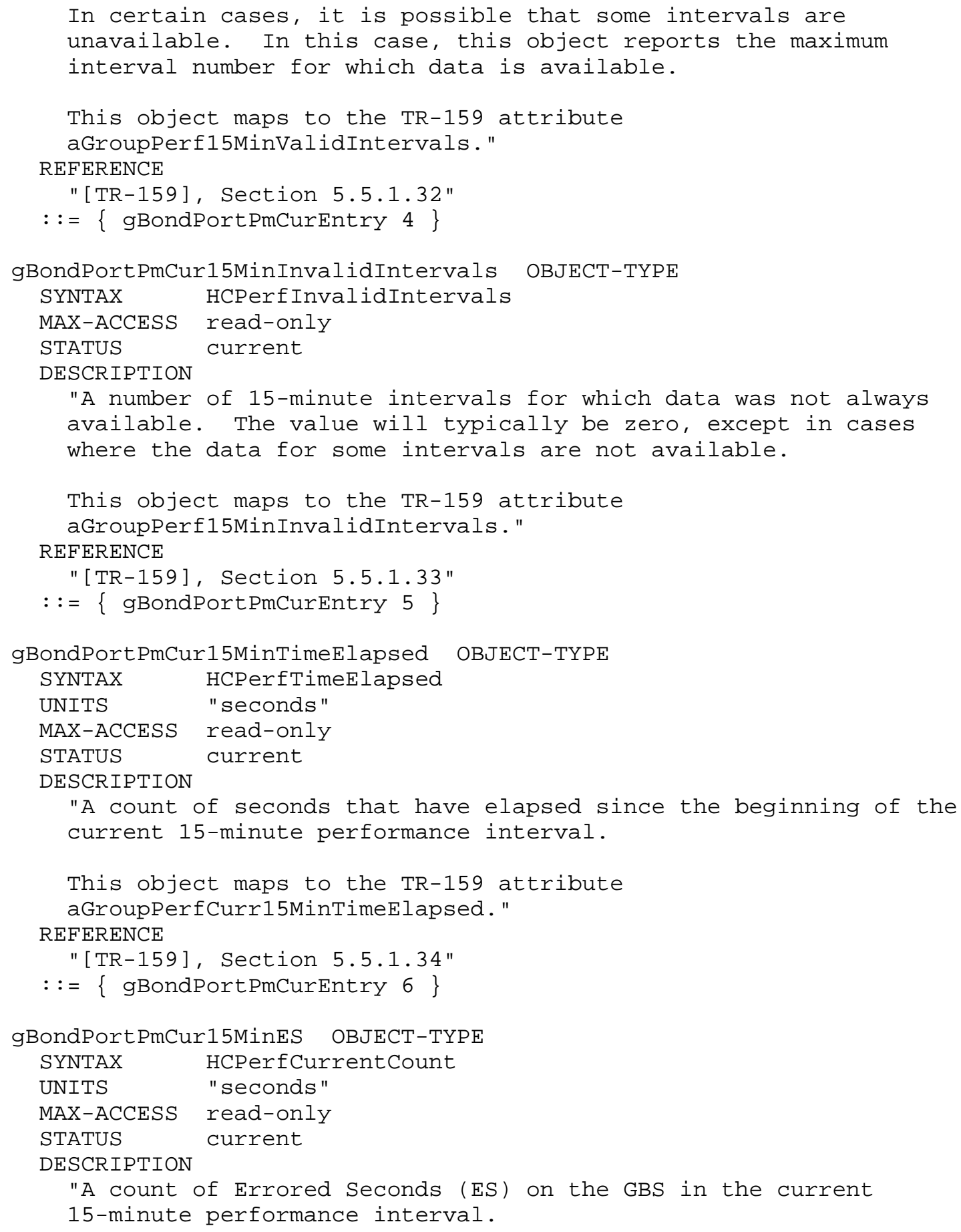




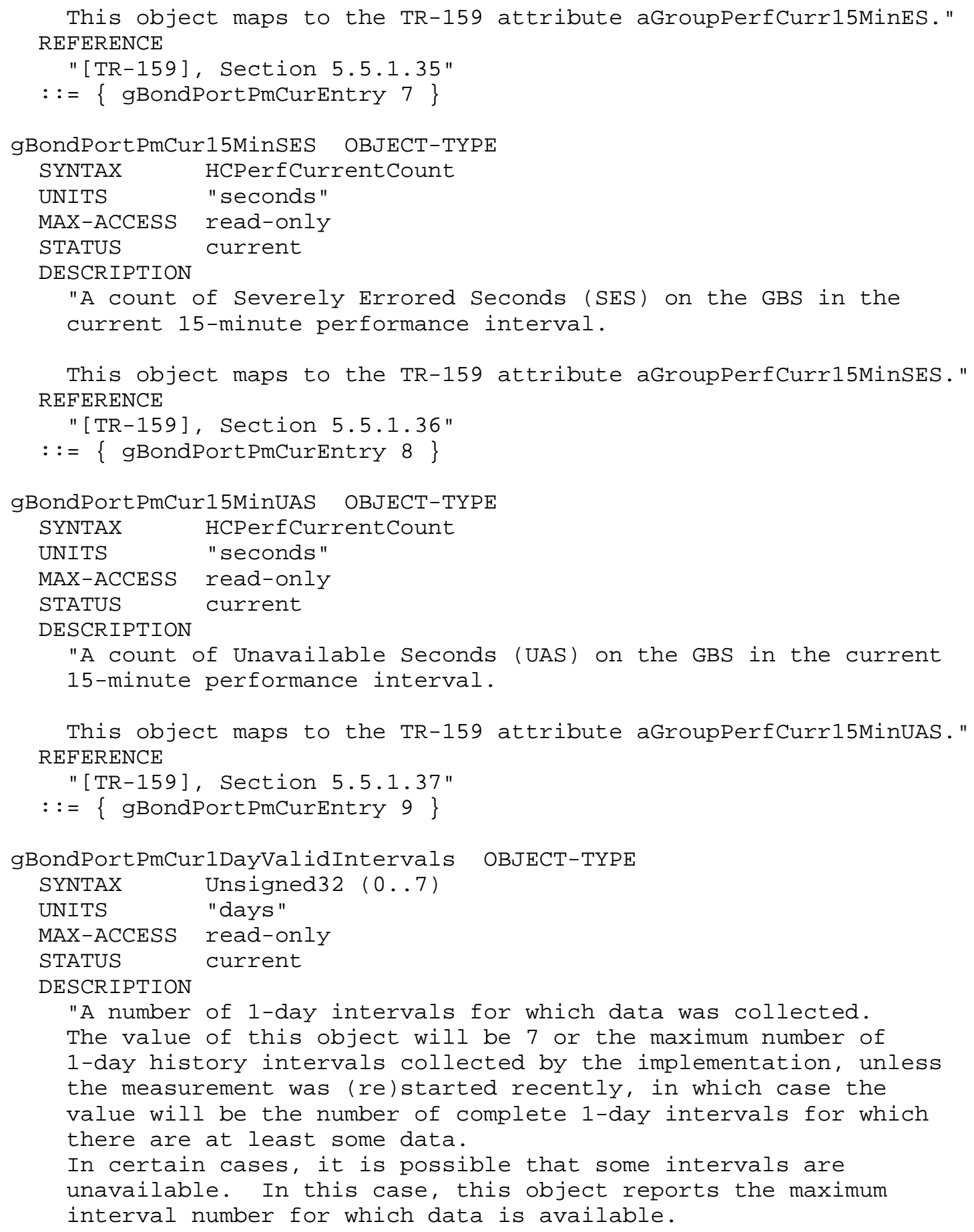




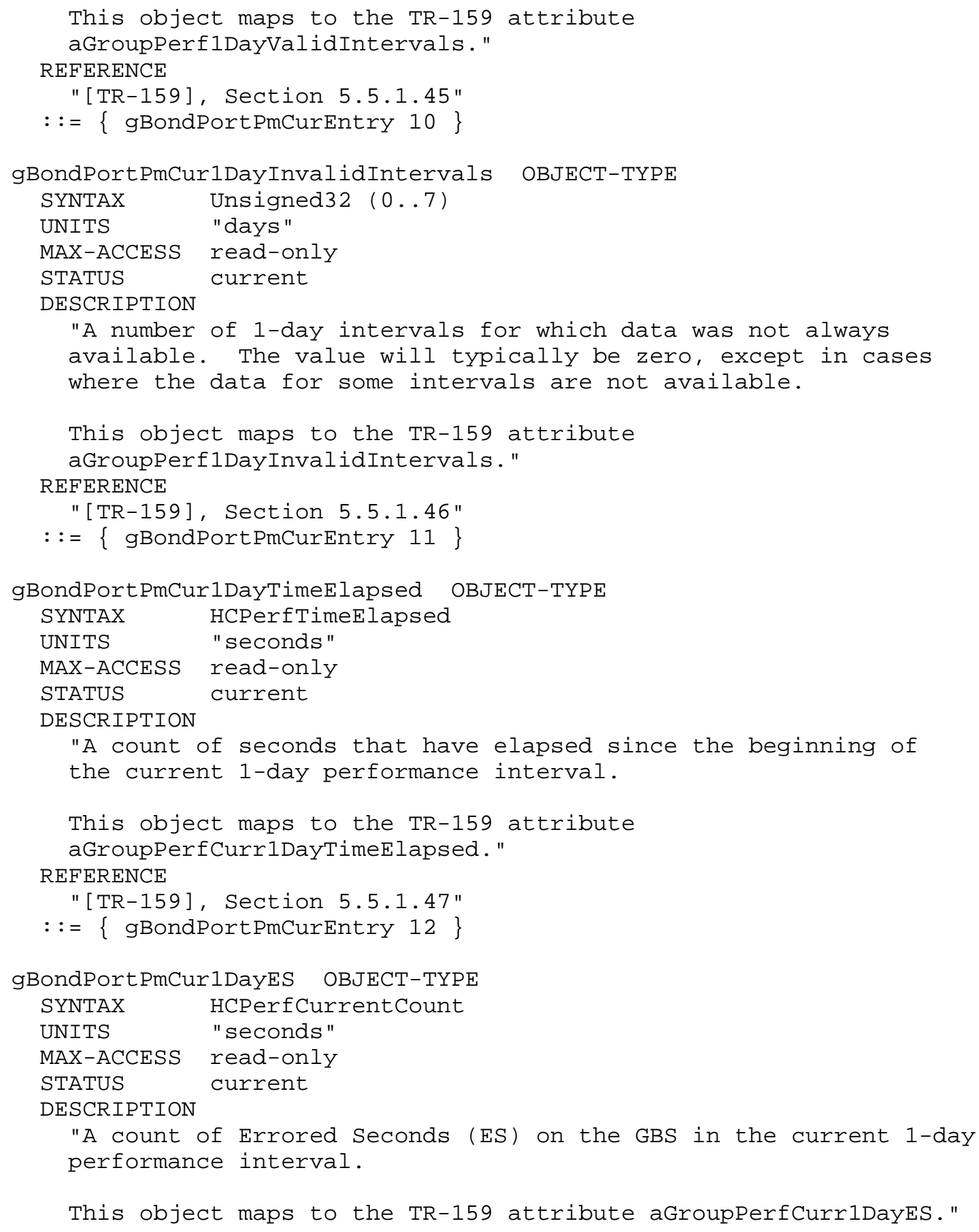




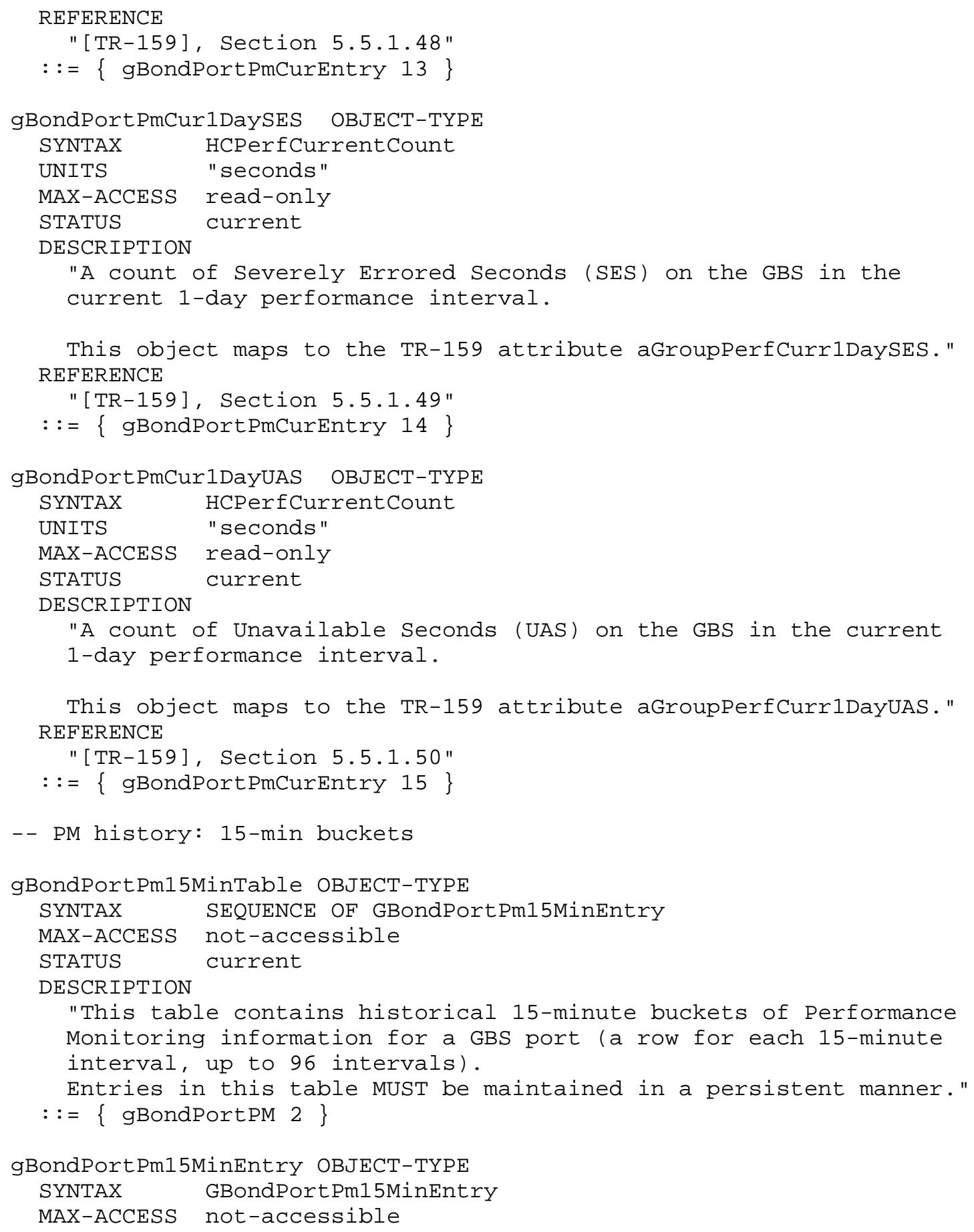




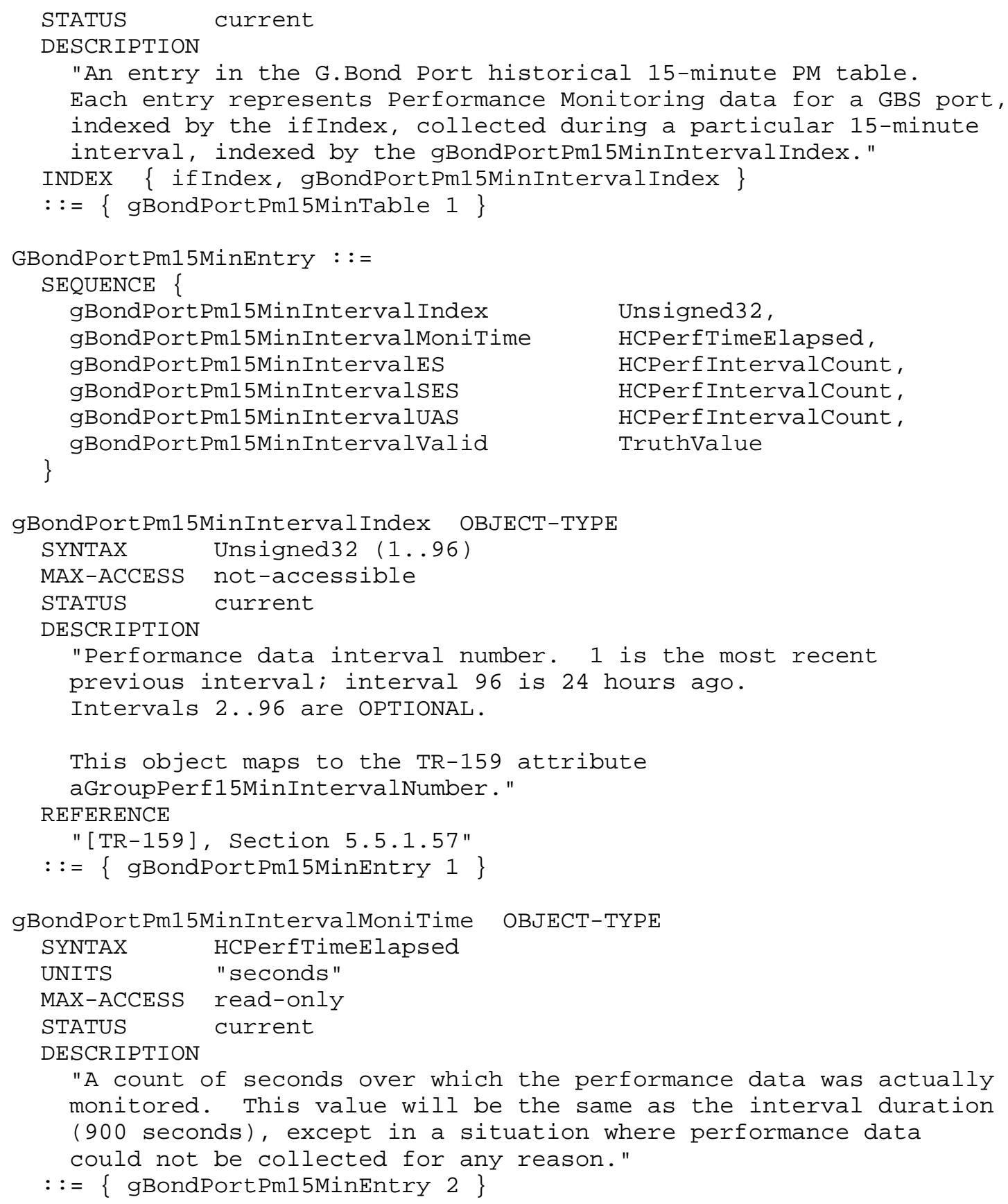




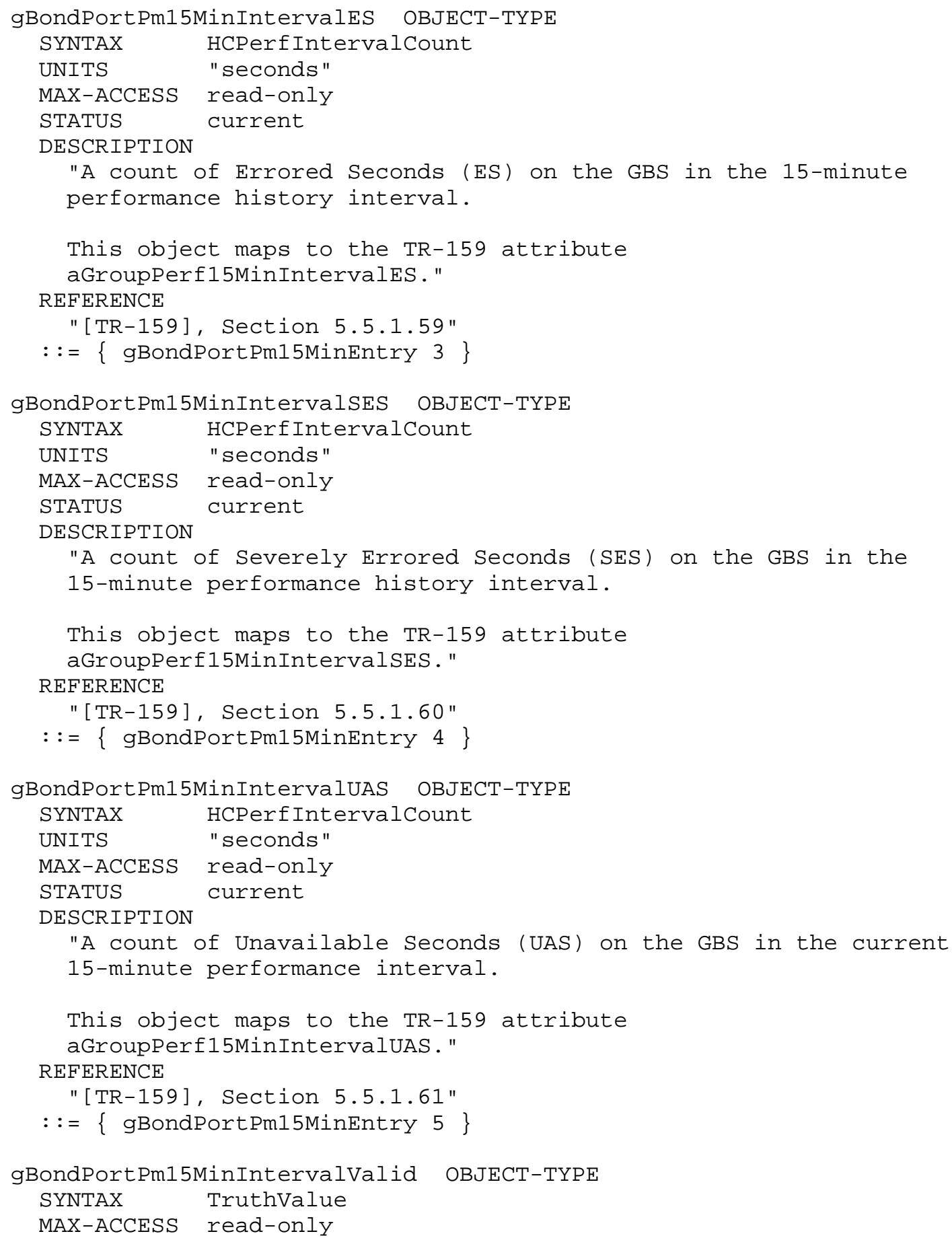




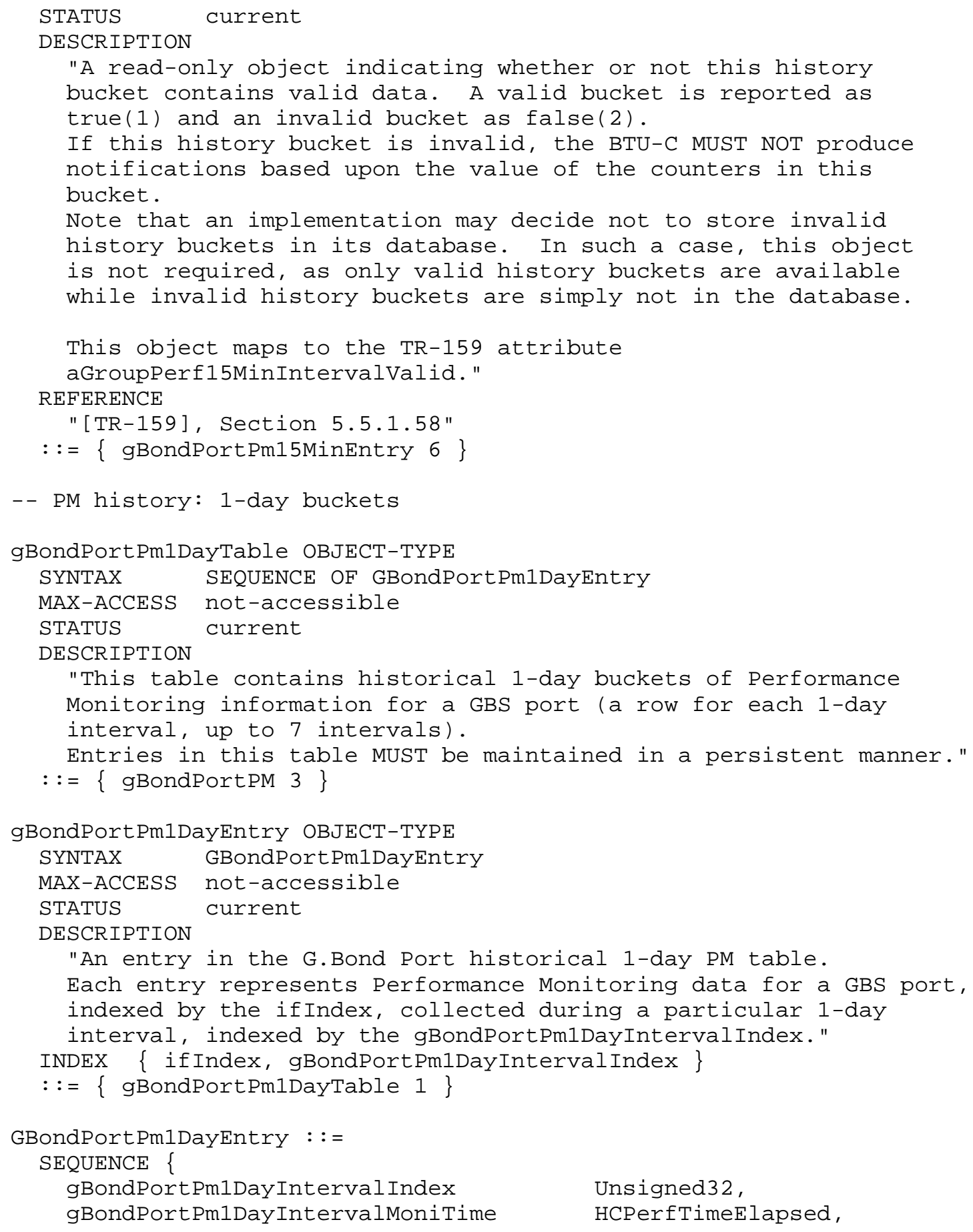




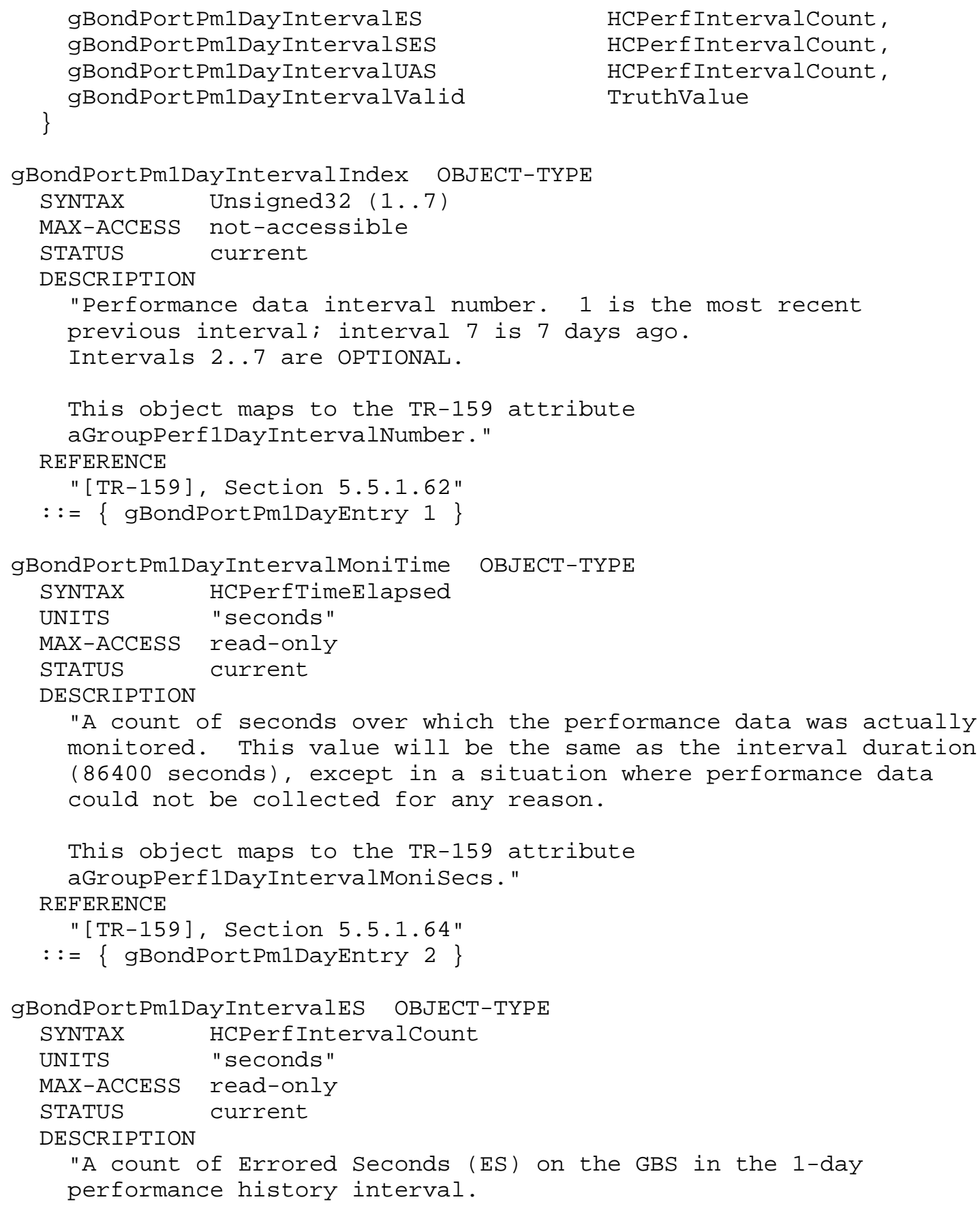




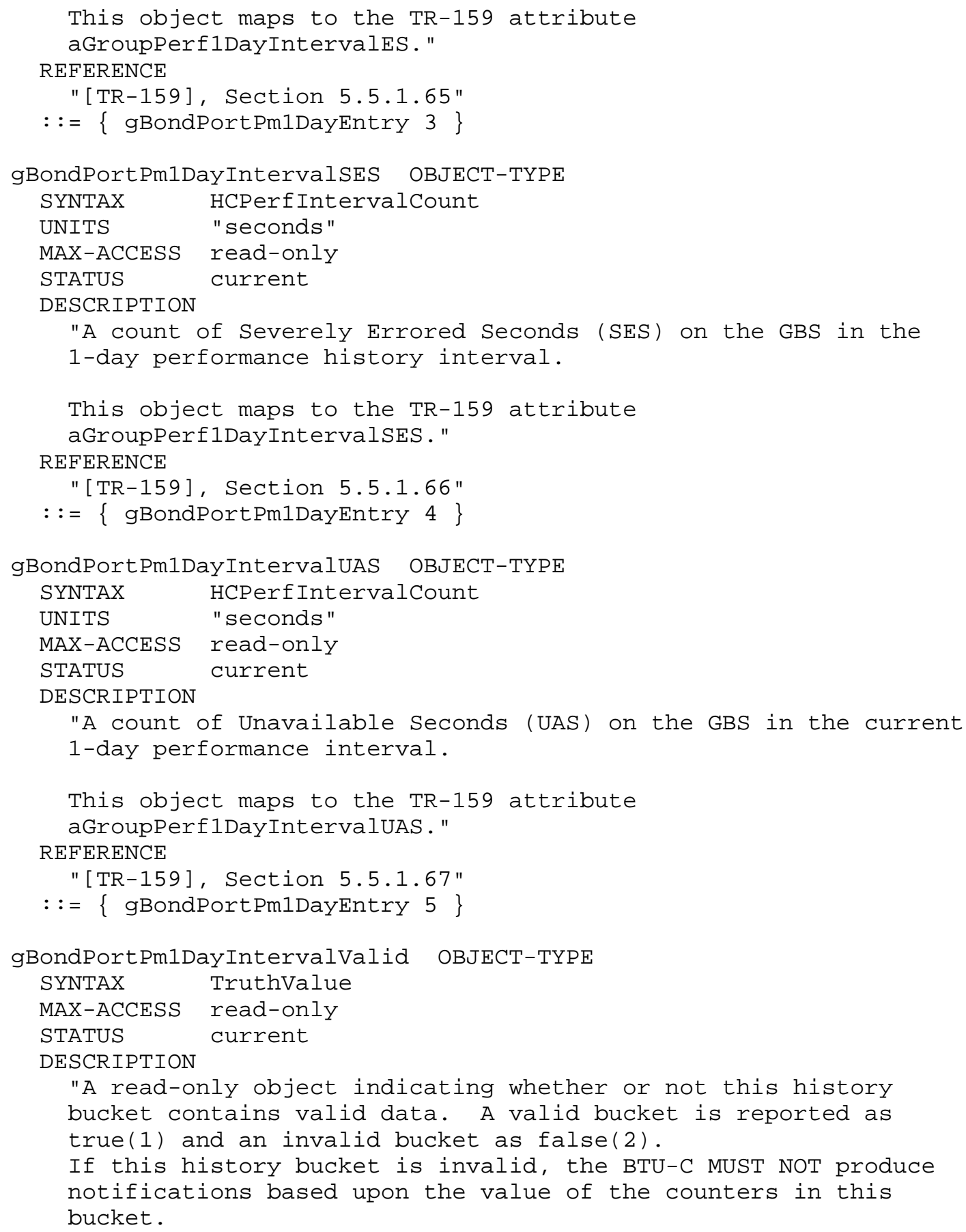




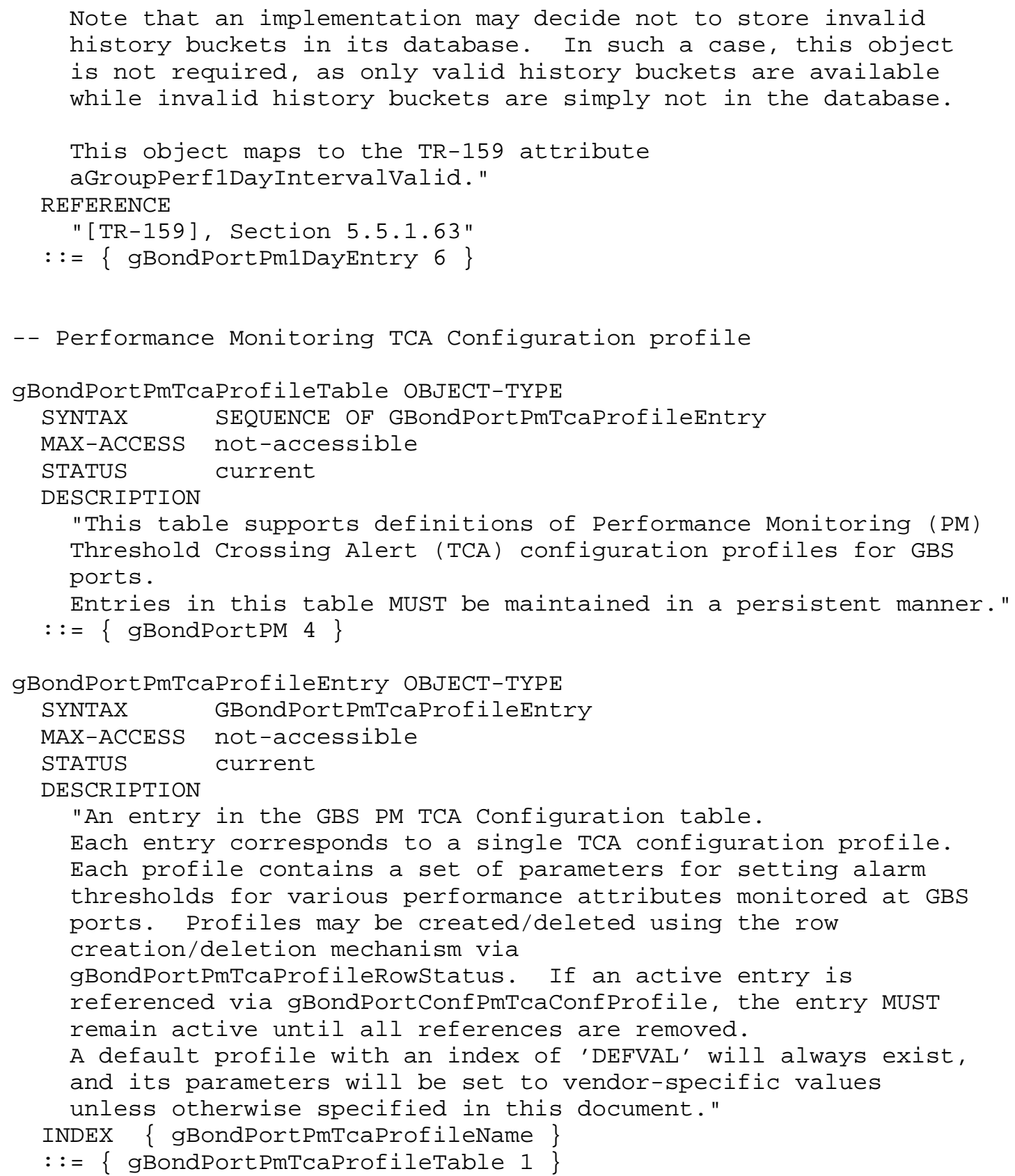




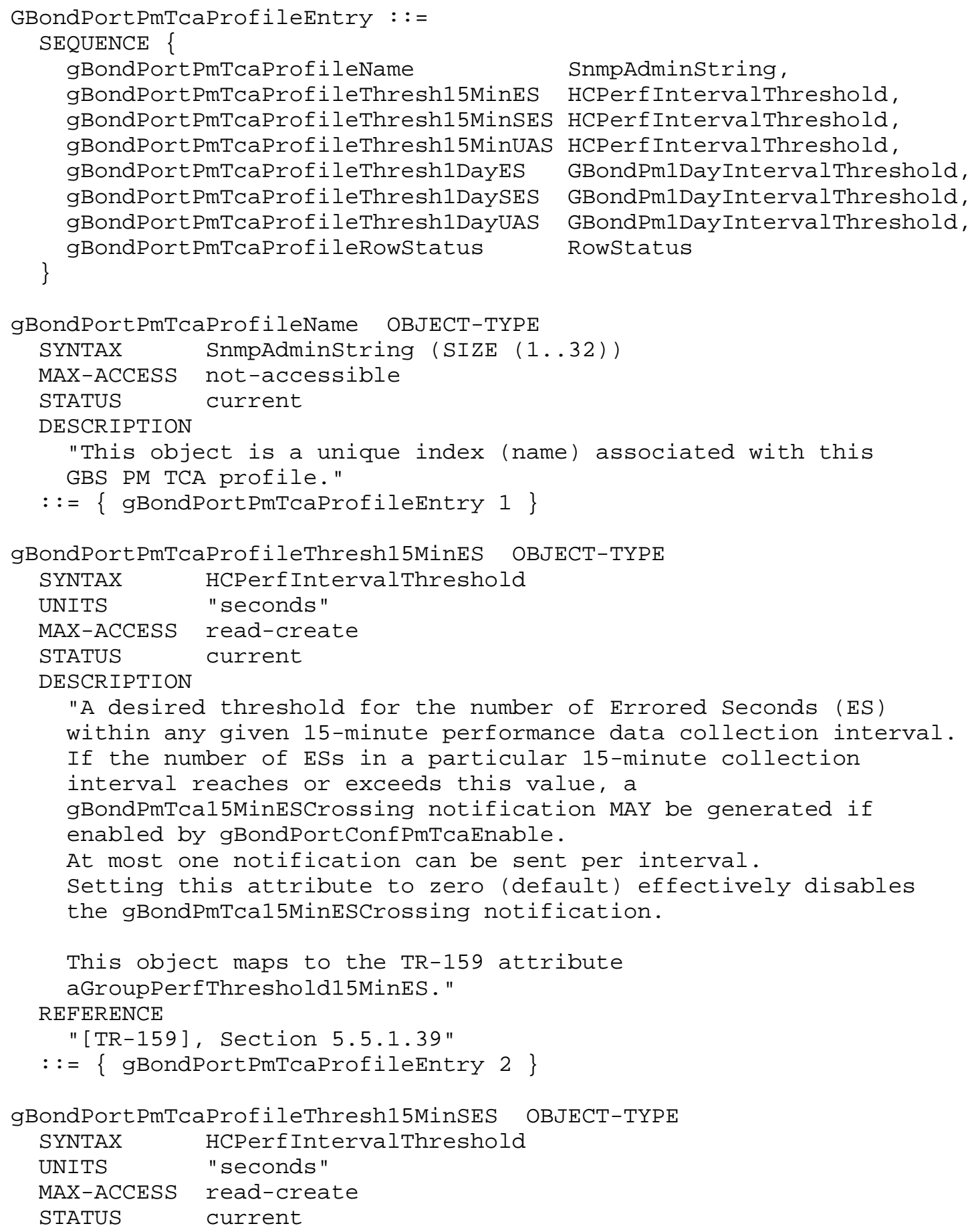




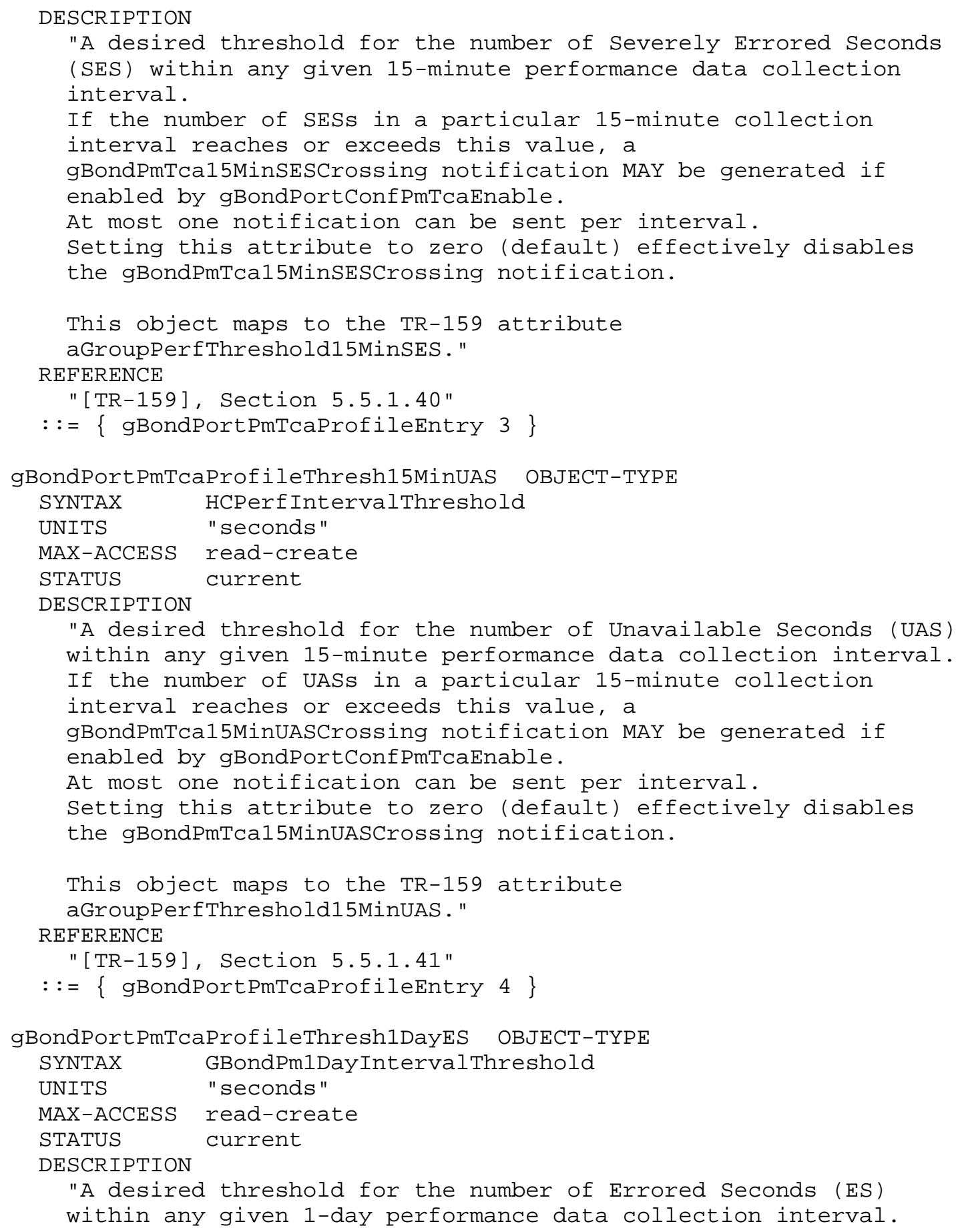


If the number of ESs in a particular 1-day collection interval reaches or exceeds this value, a gBondPmTcalDayEscrossing notification MAY be generated if enabled by gBondPortConfPmTcaEnable. At most one notification can be sent per interval. Setting this attribute to zero (default) effectively disables the gBondPmTcalDayESCrossing notification.

This object maps to the TR-159 attribute aGroupPerfThreshold1DayES." REFERENCE

"[TR-159], Section 5.5.1.51"

$::=\{$ gBondPortPmTcaProfileEntry 5$\}$

gBondPortPmTcaProfileThresh1DaySES OBJECT-TYPE

SYNTAX GBondPmlDayIntervalThreshold

UNITS "seconds"

MAX-ACCESS read-create

STATUS current

DESCRIPTION

"A desired threshold for the number of Severely Errored Seconds (SES) within any given 1-day performance data collection interval.

If the number of SESs in a particular 1-day collection interval reaches or exceeds this value, a gBondPmTcalDaysESCrossing notification MAY be generated if enabled by gBondPortConfPmTcaEnable. At most one notification can be sent per interval. Setting this attribute to zero (default) effectively disables the gBondPmTcalDaySESCrossing notification.

This object maps to the TR-159 attribute aGroupPerfThreshold1DaysES." REFERENCE

"[TR-159], Section 5.5.1.52"

$::=\{$ gBondPortPmTcaProfileEntry 6$\}$

gBondPortPmTcaProfileThresh1DayUAS OBJECT-TYPE

SYNTAX GBondPm1DayIntervalThreshold

UNITS "seconds"

MAX-ACCESS read-create

STATUS current

DESCRIPTION

"A desired threshold for the number of Unavailable Seconds (UAS) within any given 1-day performance data collection interval. If the number of UASs in a particular 1-day collection interval reaches or exceeds this value, a gBondPmTcalDayUASCrossing notification MAY be generated if enabled by 


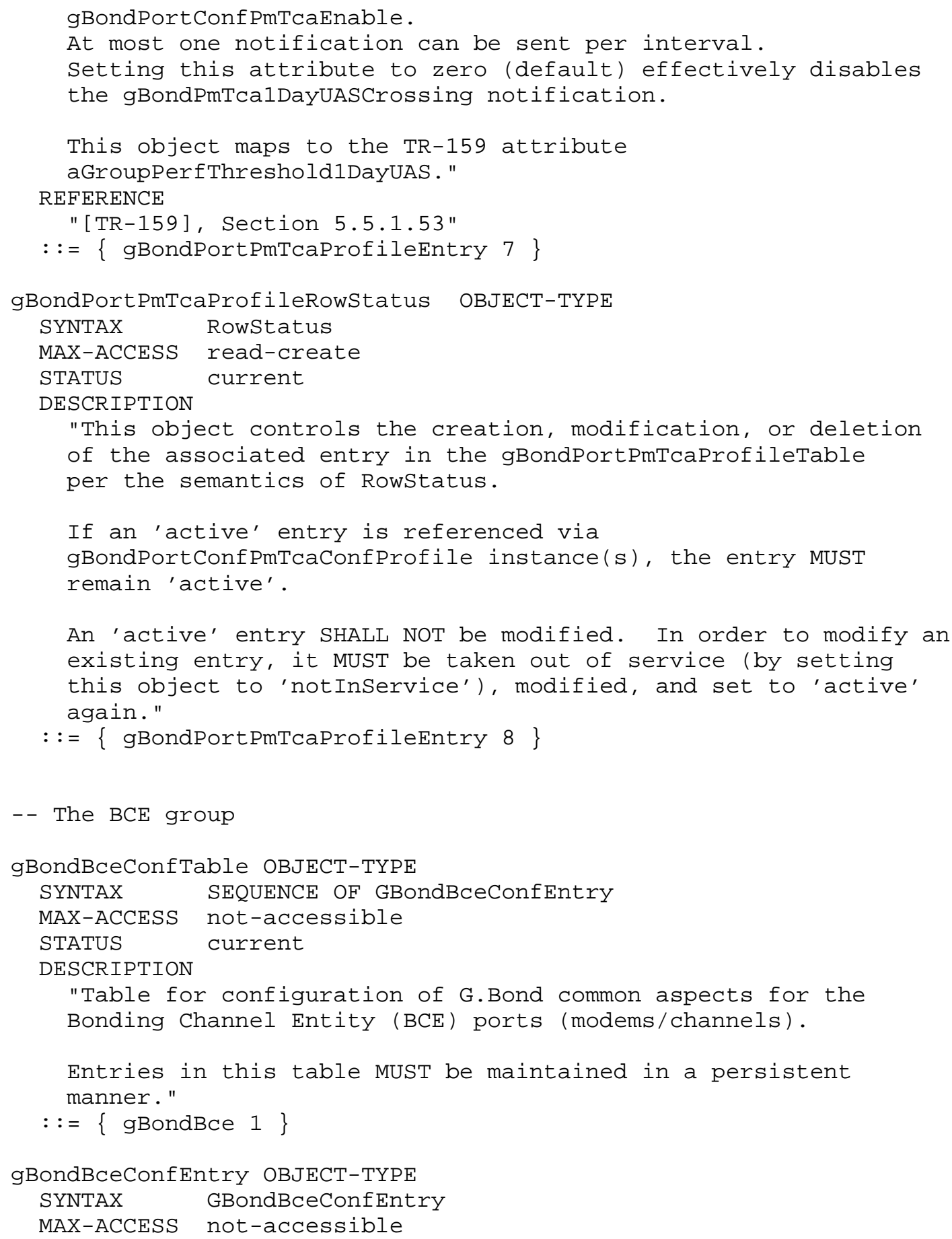




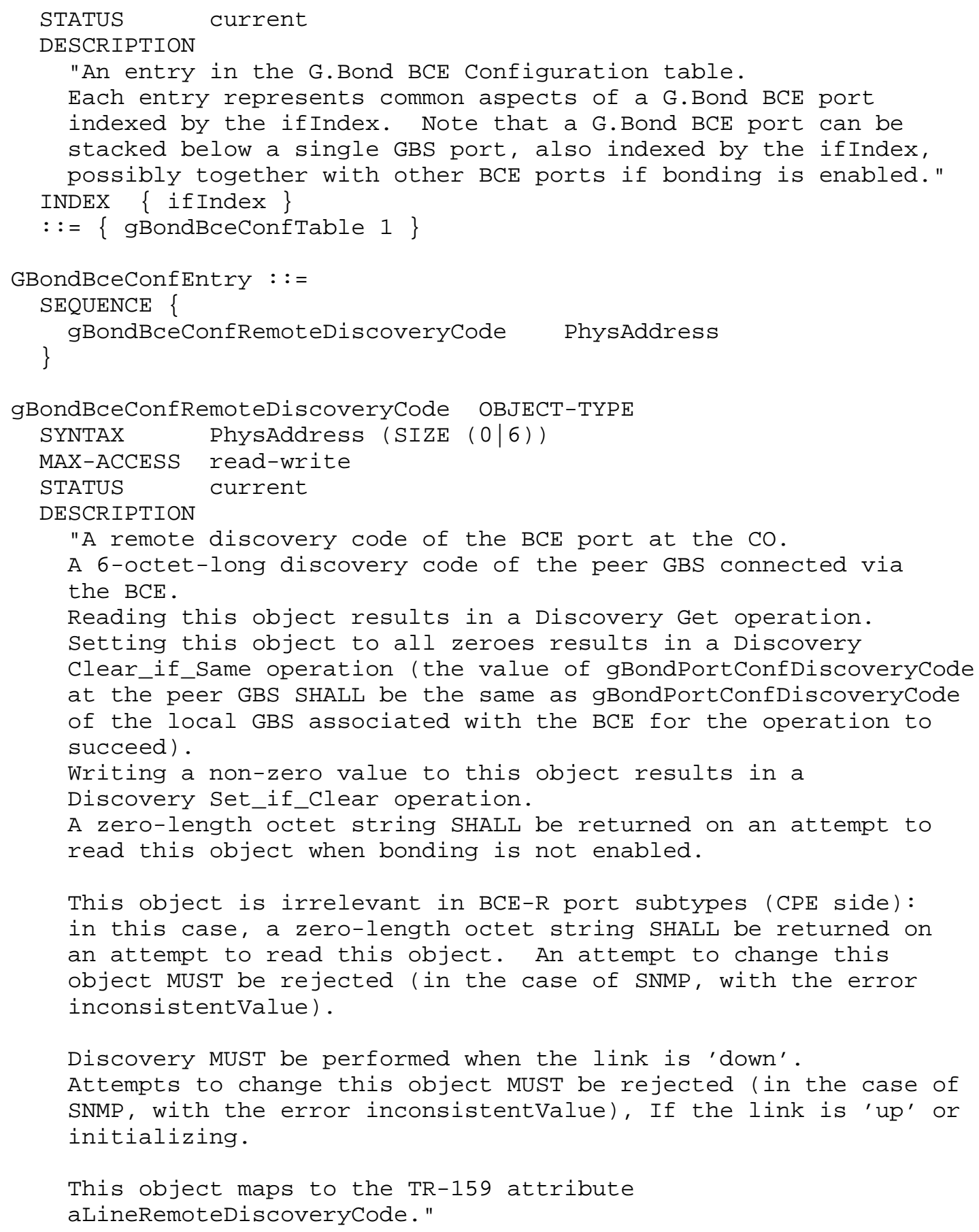




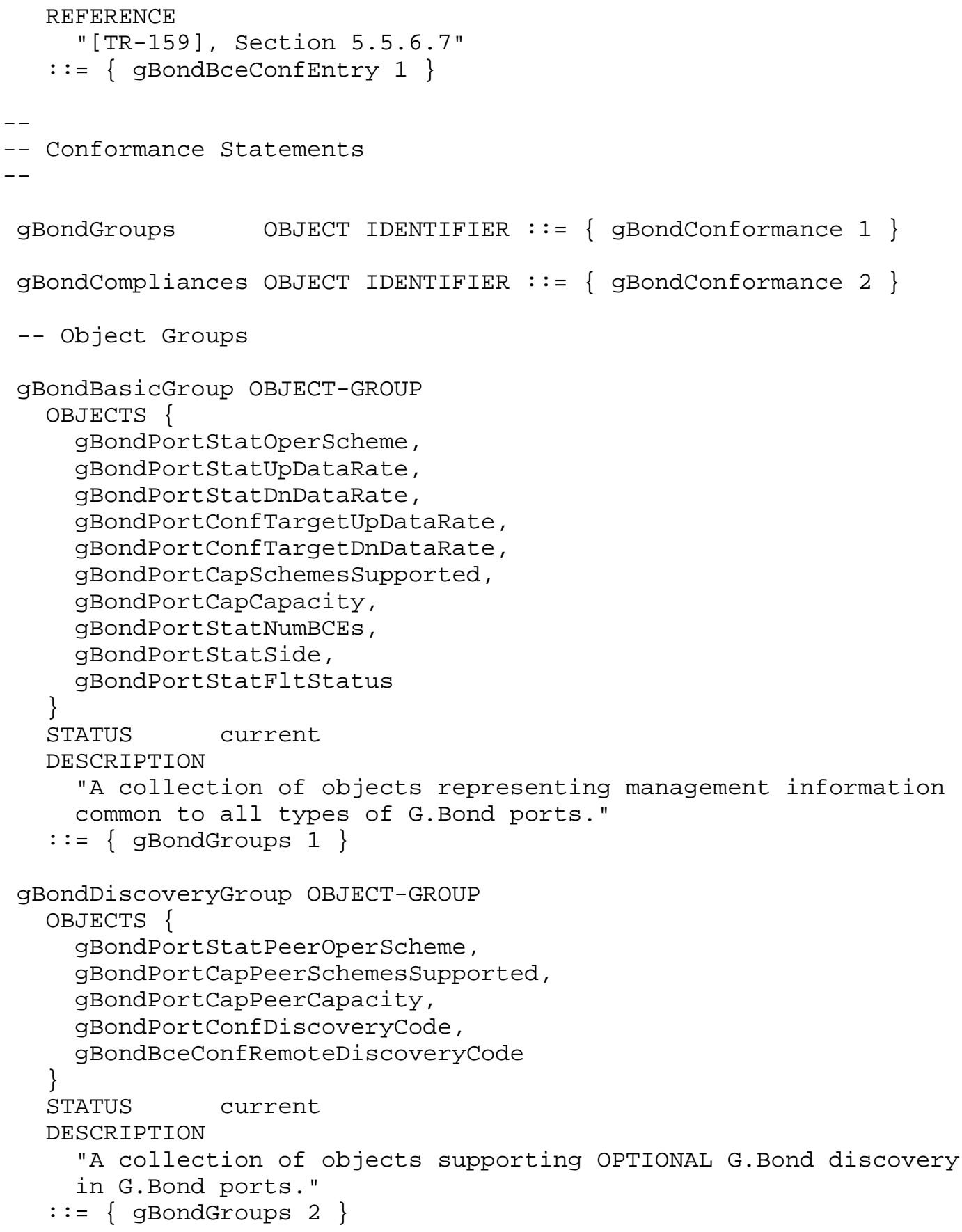

"A collection of objects supporting OPTIONAL G.Bond discovery

in G.Bond ports."

$::=\{$ gBondGroups 2$\}$ 


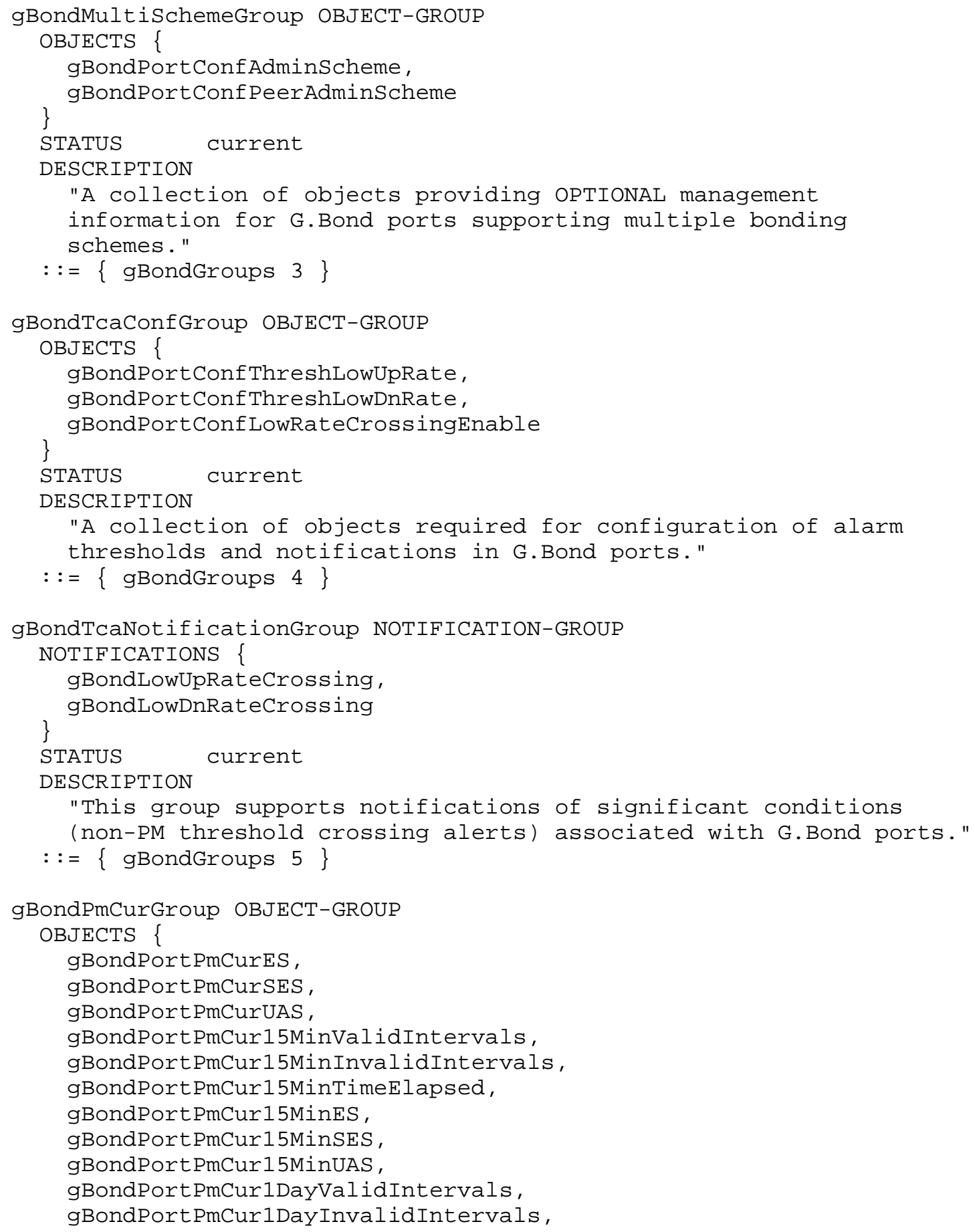




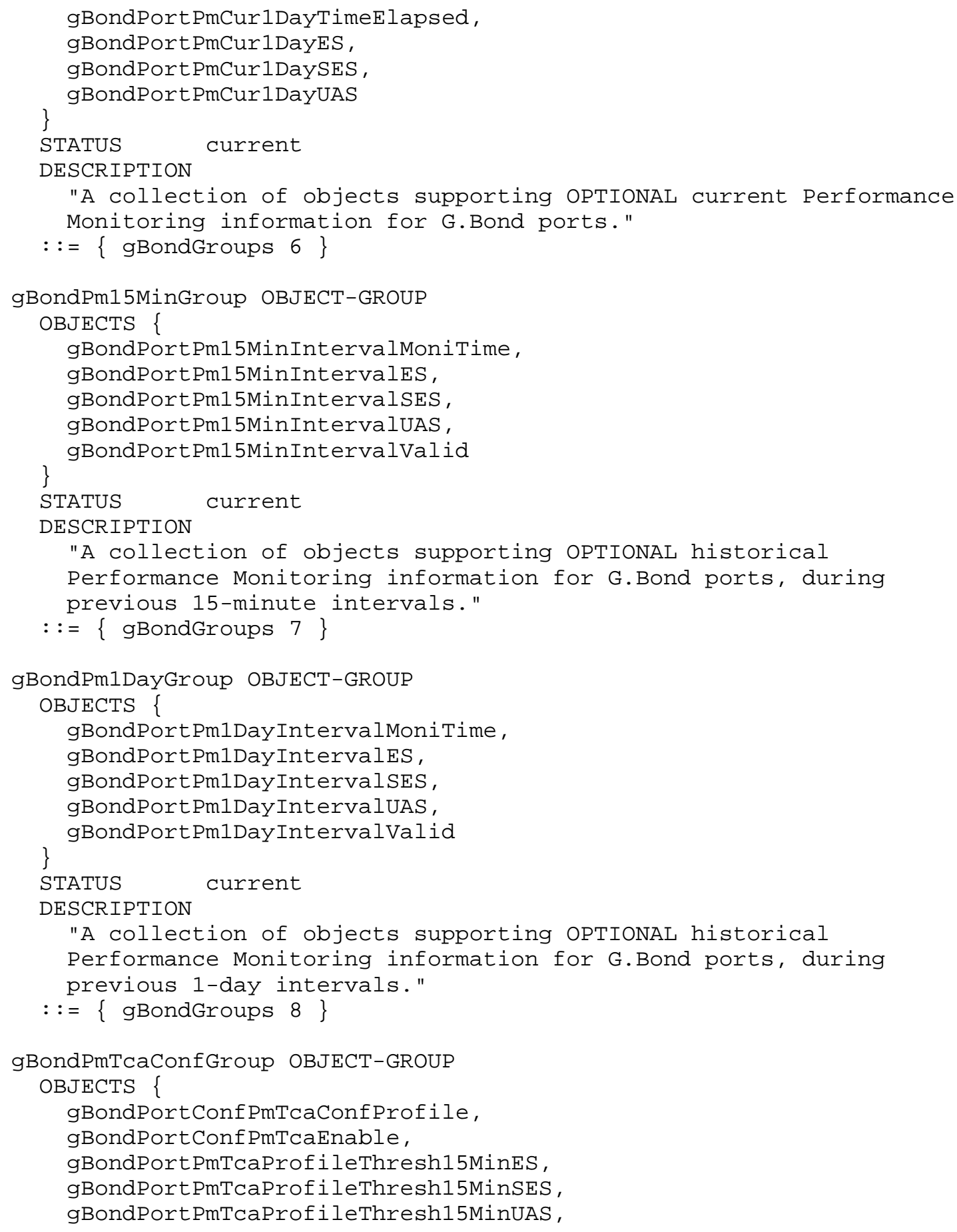

"A collection of objects supporting OPTIONAL current Performance 


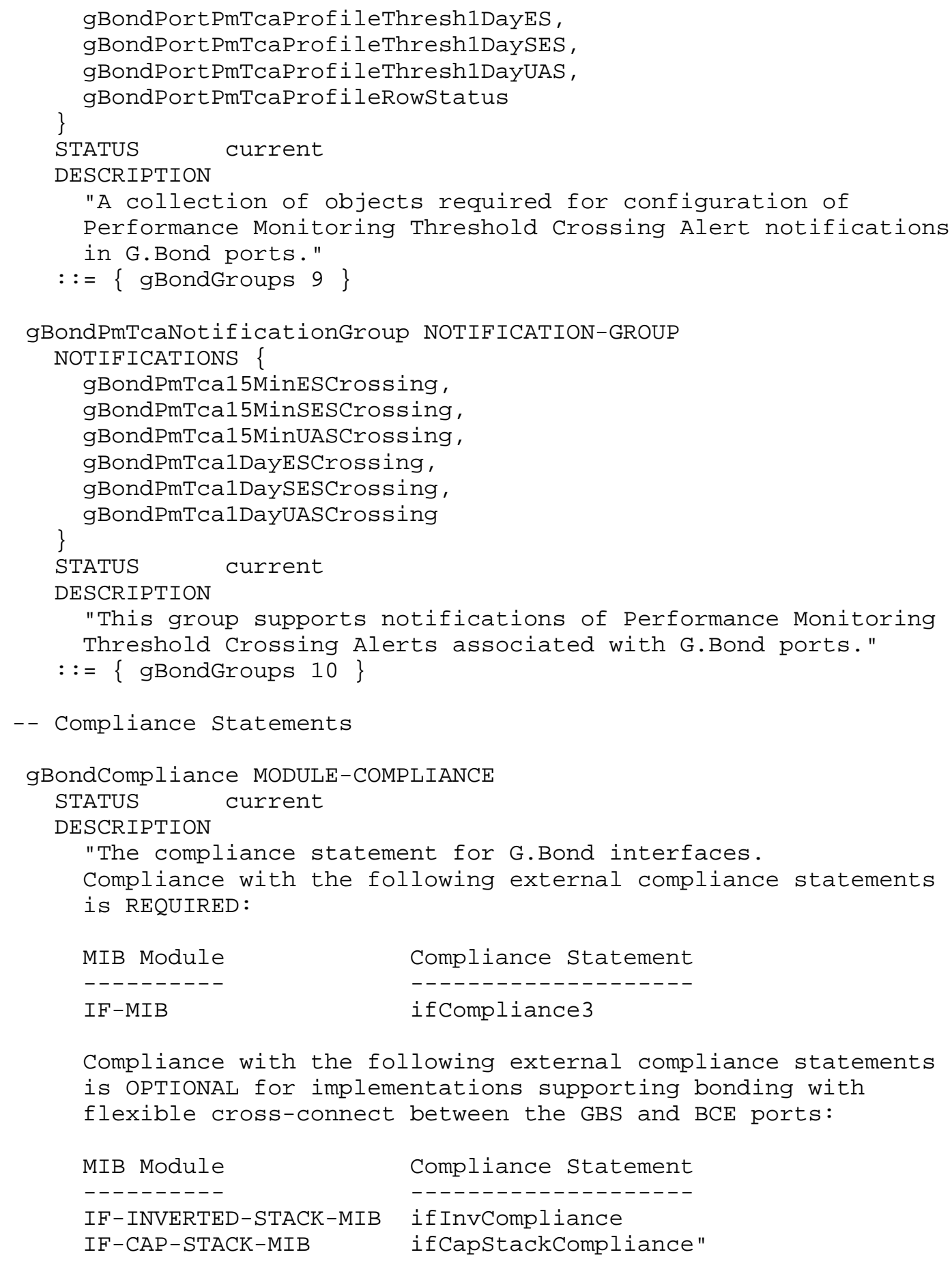




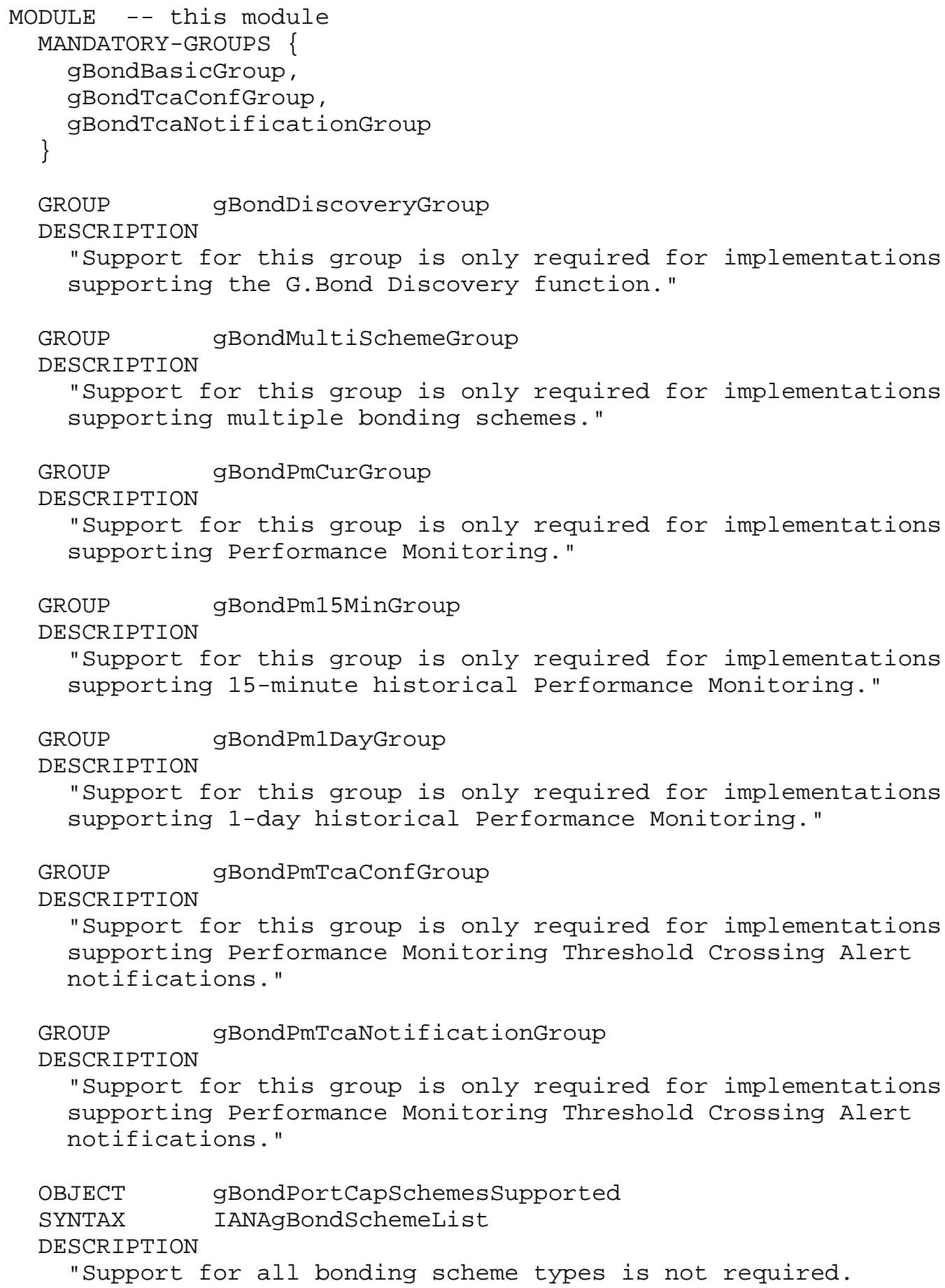




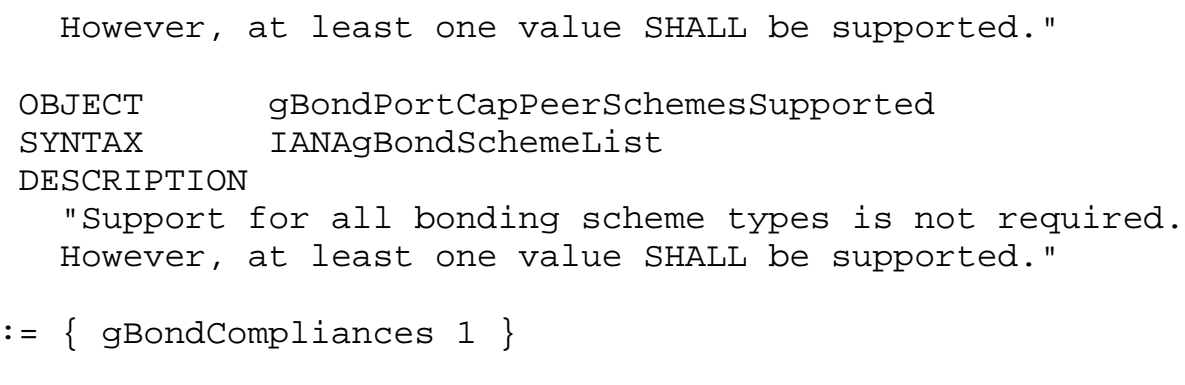


7. IANA-Maintained G.Bond TC Definitions

The IANA-GBOND-TC-MIB module IMPORTS objects from SNMPV2-SMI [RFC2578] and SNMPv2-TC [RFC2579].

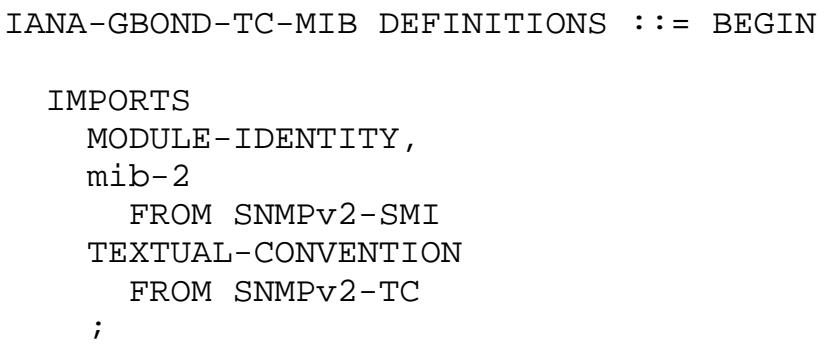


[G.998.2] refers to:

ITU-T Recommendation G.998.2: 'Ethernet-based multi-pair bonding', January 2005 .

[G.998.3] refers to:

ITU-T Recommendation G.998.3: 'Multi-pair bonding using

time-division inverse multiplexing', January 2005.

Naming Conventions:

BCE - Bonding Channel Entity

GBS - Generic Bonding Sub-layer

These references should be updated as appropriate when a new bonding scheme is added to this MIB module.

Copyright (c) 2013 IETF Trust and the persons identified as authors of the code. All rights reserved.

Redistribution and use in source and binary forms, with or without modification, is permitted pursuant to, and subject to the license terms contained in, the Simplified BSD License set forth in Section 4.c of the IETF Trust's Legal Provisions Relating to IETF Documents (http://trustee.ietf.org/license-info)."

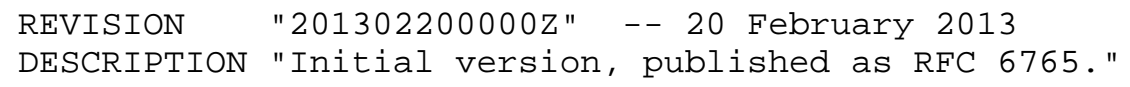




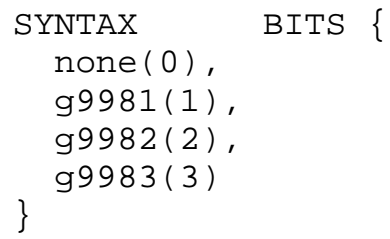

END

8. Security Considerations

There are a number of management objects defined in this MIB module with a MAX-ACCESS clause of read-write and/or read-create. Such objects may be considered sensitive or vulnerable in some network environments. The support for SET operations in a non-secure environment without proper protection can have a negative effect on network operations. These are the tables and objects and their sensitivity/vulnerability:

o Changing of the gBondPortConfAdminScheme object may lead to a potential locking of the link, if the peer device does not support the desired bonding scheme.

- Changing of the gBondPortConfDiscoveryCode object, before the discovery operation, may lead to a wrongful discovery -- for example, when two CO ports are connected to the same multi-channel $\mathrm{RT}$ port, while both $\mathrm{CO}$ ports have the same discovery register value. 
- Changing of the target upstream/downstream data rate via gBondPortConfTargetUpDataRate/gBondPortConfTargetDnDataRate may lead to anything from degradation of link quality and data rate to a complete link initialization failure, as the ability of a G.Bond port to support a particular configuration depends on the copper environment.

- Activation of a specific line/channel may cause a severe degradation of service for another G.Bond port, whose channel(s) may be affected by the cross-talk from the newly activated channel.

- Removal of a channel from an operationally 'up' G.Bond port, aggregating several channels, may cause degradation of the port's data rate.

Some of the readable objects in this MIB module (i.e., those with MAX-ACCESS other than not-accessible) may be considered sensitive or vulnerable in some network environments since, collectively, they provide information about the performance of network interfaces and can reveal some aspects of their configuration.

In particular, since a bonded xDSL port can be comprised of multiple Unshielded Twisted Pair (UTP) voice-grade copper, located in the same bundle with other pairs belonging to another operator/customer, it is theoretically possible to eavesdrop on a G.Bond transmission, simply by "listening" to cross-talk from the bonded pairs, especially if the operating parameters of the G.Bond link in question are known.

It is thus important to control even GET and/or NOTIFY access to these objects and possibly to even encrypt the values of these objects when sending them over the network via SNMP. These are the tables and objects and their sensitivity/vulnerability:

- gBondPortstatTable - objects in this table provide status information for the G.Bond port, which may aid in identification of the pairs belonging to the bonded port and eavesdropping on the traffic over that port.

SNMP versions prior to SNMPv3 did not include adequate security. Even if the network itself is secure (for example by using IPsec), there is no control as to who on the secure network is allowed to access and GET/SET (read/change/create/delete) the objects in this MIB module.

Implementations SHOULD provide the security features described by the SNMPv3 framework (see [RFC3410]), and implementations claiming compliance to the SNMPv3 standard MUST include full support for 
authentication and privacy via the User-based Security Model (USM) [RFC3414] with the AES cipher algorithm [RFC3826]. Implementations MAY also provide support for the Transport Security Model (TSM) [RFC5591] in combination with a secure transport such as SSH [RFC5592] or TLS/DTLS [RFC6353].

Further, deployment of SNMP versions prior to SNMPv3 is NOT RECOMMENDED. Instead, it is RECOMMENDED to deploy SNMPv3 and to enable cryptographic security. It is then a customer/operator responsibility to ensure that the SNMP entity giving access to an instance of this MIB module is properly configured to give access to the objects only to those principals (users) that have legitimate rights to indeed GET or SET (change/create/delete) them.

9. IANA Considerations

Three new values of IANAiftype -- g9981(263), g9982(264), and g9983 (265) -- have been allocated by IANA <http://www.iana.org/> in the IANAifType-MIB module [IANAifType-MIB].

An object identifier for gBondMIB MODULE-IDENTITY has been allocated by IANA in the MIB-2 transmission sub-tree (211).

This document defines the first version of the IANA-maintained IANAGBOND-TC-MIB module. It is intended that each new G.998 bonding scheme defined by the ITU-T Q4/SG15 working group and approved for publication in a revision of ITU-T G.998.x will be added to the IANAmaintained MIB module, provided that it is suitable for being managed by the base objects in the GBOND-MIB module. An object identifier for ianaGBondTCMIB MODULE-IDENTITY has been allocated by IANA in the MIB-2 transmission sub-tree (215).

For each new bonding scheme added, a short description of the bonding protocol and, wherever possible, a reference to a publicly available specification SHOULD be specified. An Expert Review, as defined in [RFC5226], is REQUIRED for each modification.

\section{Acknowledgments}

This document was produced by the [ADSLMIB] working group. Special thanks to Dan Romascanu for his meticulous review of this text. 


\title{
11. References
}

\subsection{Normative References}

\author{
[RFC2119] Bradner, S., "Key words for use in RFCs to Indicate \\ Requirement Levels", BCP 14, RFC 2119, March 1997. \\ [RFC2578] McCloghrie, K., Ed., Perkins, D., Ed., and J. \\ Schoenwaelder, Ed., "Structure of Management Information \\ Version 2 (SMIv2)", STD 58, RFC 2578, April 1999. \\ [RFC2579] MCCloghrie, K., Ed., Perkins, D., Ed., and J. \\ Schoenwaelder, Ed., "Textual Conventions for SMIv2", \\ STD 58, RFC 2579, April 1999. \\ [RFC2580] McCloghrie, K., Perkins, D., and J. Schoenwaelder, \\ "Conformance Statements for SMIv2", STD 58, RFC 2580, \\ April 1999. \\ [RFC2863] McCloghrie, K. and F. Kastenholz, "The Interfaces Group \\ MIB", RFC 2863, June 2000 . \\ [RFC3411] Harrington, D., Presuhn, R., and B. Wijnen, "An \\ Architecture for Describing Simple Network Management \\ Protocol (SNMP) Management Frameworks", STD 62, RFC 3411, \\ December 2002 . \\ [RFC3414] Blumenthal, U. and B. Wijnen, "User-based Security Model \\ (USM) for version 3 of the Simple Network Management \\ Protocol (SNMPv3)", STD 62, RFC 3414, December 2002. \\ [RFC3705] Ray, B. and R. Abbi, "High Capacity Textual Conventions \\ for MIB Modules Using Performance History Based on 15 \\ Minute Intervals", RFC 3705, February 2004. \\ [RFC3826] Blumenthal, U., Maino, F., and K. McCloghrie, "The \\ Advanced Encryption Standard (AES) Cipher Algorithm in the \\ SNMP User-based Security Model", RFC 3826, June 2004. \\ [RFC5226] Narten, T. and H. Alvestrand, "Guidelines for Writing an \\ IANA Considerations section in RFCs", BCP 26, RFC 5226, \\ May 2008 . \\ [RFC5591] Harrington, D. and W. Hardaker, "Transport Security Model \\ for the Simple Network Management Protocol (SNMP)", \\ RFC 5591, June 2009.
}


[RFC5592] Harrington, D., Salowey, J., and W. Hardaker, "Secure Shell Transport Model for the Simple Network Management Protocol (SNMP)", RFC 5592, June 2009.

[RFC6353] Hardaker, W., "Transport Layer Security (TLS) Transport Model for the Simple Network Management Protocol (SNMP)", RFC 6353, July 2011.

[TR-159] Beili, E. and M. Morgenstern, "Management Framework for xDSL Bonding", Broadband Forum Technical Report TR-159, December 2008, <http://www.broadband-forum.org/technical/ download/TR-159.pdf>.

11.2. Informative References

[802.3] IEEE, "IEEE Standard for Information technology Telecommunications and information exchange between systems - Local and metropolitan area networks - Specific requirements - Part 3: Carrier Sense Multiple Access with Collision Detection (CSMA/CD) Access Method and Physical Layer Specifications", IEEE Std 802.3-2005, December 2005.

[ADSLMIB] IETF, "ADSL MIB (adslmib) Charter", <http://datatracker.ietf.org/wg/adslmib/charter/>.

$[\mathrm{AF}-\mathrm{PHY}-0086]$

ATM Forum, "Inverse Multiplexing for ATM (IMA) Specification Version 1.1", ATM Forum specification afphy-0086.001, March 1999, <http://www.broadband-forum.org/ $\mathrm{ftp} / \mathrm{pub} /$ approved-specs/af-phy-0086.001.pdf>.

[G.994.1] ITU-T, "Handshake procedures for digital subscriber line (DSL) transceivers", ITU-T Recommendation G.994.1, June 2012, <http://www.itu.int/rec/T-REC-G.994.1/en>.

[G.998.1] ITU-T, "ATM-based multi-pair bonding", ITU-T Recommendation G.998.1, January 2005, <http://wWw.itu.int/rec/T-REC-G.998.1/en>.

[G.998.2] ITU-T, "Ethernet-based multi-pair bonding", ITU-T Recommendation G.998.2, January 2005, <http://wWw.itu.int/rec/T-REC-G.998.2/en>.

[G.998.3] ITU-T, "Multi-pair bonding using time-division inverse multiplexing", ITU-T Recommendation G.998.3, January 2005, <http://www.itu.int/rec/T-REC-G.998.3/en>. 


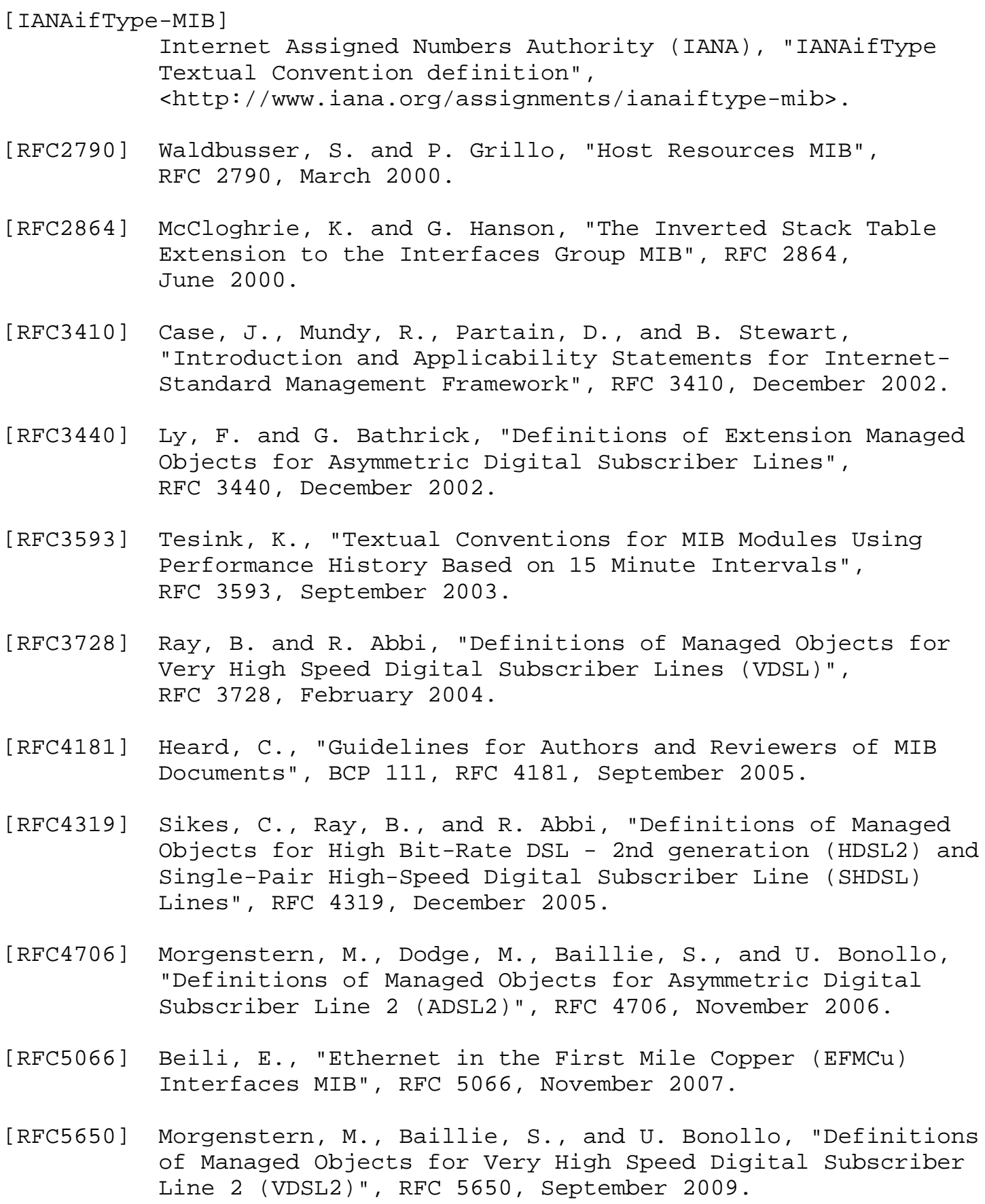


[RFC5905] Mills, D., Martin, J., Burbank, J., and W. Kasch, "Network Time Protocol Version 4: Protocol and Algorithms Specification", RFC 5905, June 2010.

[RFC6766] Beili, E., "xDSL Multi-Pair Bonding Using Time-Division Inverse Multiplexing (G.Bond/TDIM) MIB", RFC 6766, February 2013 .

[RFC6767] Beili, E. and M. Morgenstern, "Ethernet-Based xDSL MultiPair Bonding (G.Bond/Ethernet) MIB", RFC 6767, February 2013 .

[RFC6768] Beili, E., "ATM-Based xDSL Bonded Interfaces MIB", RFC 6768, February 2013.

Authors' Addresses

Edward Beili

Actelis Networks

25 Bazel St.

Petach-Tikva 49103

Israel

Phone: +972-3-924-3491

EMail: edward.beili@actelis.com

Moti Morgenstern

ECI Telecom

30 Hasivim St.

Petach-Tikva 4951169

Israel

Phone: +972-3-926-6258

EMail: moti.morgenstern@ecitele.com 